\title{
A Robust Method for Diagnostic Energetic System with Bond Graph
}

\author{
Belgacem Hamdouni ${ }^{1}$, Dhafer Mezghani ${ }^{2}$, Jamel Riahi ${ }^{3}$, Abdelkader Mami ${ }^{4}$ \\ UR-LAPER, Faculty of Sciences of Tunis, University of Tunis ElManar \\ Tunis, Tunisia
}

\begin{abstract}
Surveillance and supervision systems have a major role in insuring the safety and availability of industrial equipments and installations. Default detection and diagnosis is highly important to facilitate the planning and implementation of curative and preventive actions. Industrial systems are usually governed by different physical phenomena's and diverse technological components. Bond graph, being a powerful tool based on energetic and multi-physical analysis can be a welladapted tool in default detection. The resulting Bond Graph model, allows to apply model based diagnosis methods to detect and eventually isolate defaults. In this paper, energetic systems diagnosis problems are discussed by detailing existing diagnosis methods. The proposed modeling tool is then introduced with illustration of different use cases and applications examples. Diagnosis methods based on Bond Graph model are presented, as well as the extension of these methods with uncertain parameters models. Finally, the studied diagnosis method is applied for default detection and isolation using the study case of asynchrony motor.
\end{abstract}

Keywords-Bond graph; diagnostic; fault detection; energy systems

\section{INTRODUCTION}

Any failure of a process is harmful in an environment where performance is paramount. It is, therefore, necessary to ensure permanently the functioning vis-à-vis process goals that have been assigned. The information to translate the behavior of a system is given by the actions of one variable. The quality of measurements is essential to allow monitoring and evaluation of the performance of a process. The quality of information can be increased by improving the precision instrumentation and multiplying the number of sensors. For technical or financial reasons, this solution in which the same quantity is measured by several sensors is for high-tech industries or those with high technological risks. Furthermore, this hardware redundancy does not protect against a failure of some common elements of the electrode: several sensors measuring the same size are generally geographically neighboring and powered by the same electric network; a power failure causes a stop of the whole measurement system.

Exploiting a priori models accurate linking different measured variables is another way to check the reliability of the measurements. The analytical redundancy has the advantage of not increasing the cost of the installation and to disengage from material constraints. In the field of diagnostics, methods based on the concept of redundancy of information have been developed.

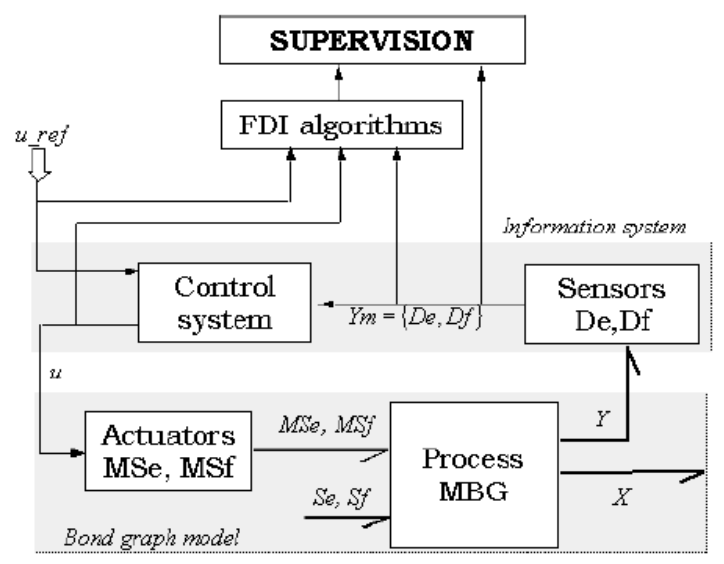

Fig. 1. Bond Graph Representation of a Monitoring System.

The mathematical process, analytical redundancy methods, therefore, require a model of the system being monitored. This model includes several parameters whose values are assumed to be known during normal operation. The comparison between the actual system behavior and the expected behavior given by the model provides a quantity called residue, which will be used to determine if the system is in a failed state or not and specify if any part or component failing system. Diagnoses of operation of a system are to detect and locate the internal defects (affecting the process itself), external faults (affecting actuators, sensors), abnormal operation, then to estimate the main characteristics of the highlighted defects (their amplitudes).

The modeling of the different sections of the pumping system that we will describe its diagnostic in this work was presented and investigated in [1].

\section{DiAgnOSTIC SYSTEM's DESCRIPTION}

The systems designed and manufactured by man (vehicles, aircraft, telecommunications networks, factories) are becoming more complex. This complexity is due to a large number of components making up these systems. Despite the high-security requirements, reducing operating costs and control of equipment availability, these systems are not immune to failure. This is why monitoring, diagnosis (detection, location, identification of failures), repairing or reconfiguration is very important. These activities help to detect and locate faults, minimize repair time and provide a reliable and easy interpret diagnosis despite the complexity of the equipment. Nowadays, the implementation of automated systems requires the establishment of important tools for the 
diagnosis and monitoring to help companies in their constant search for better functioning of their lower-cost systems. In this context, many approaches have been developed for fault detection and diagnosis by different auto research communities. These approaches can be classified generally as methods based models, pattern recognition basis, based on Bayesian networks, base case, and methods based decision tree. The model-based methods consider a behavioral model based on fundamental physical principles. These models can be of the quantitative type expressed as mathematical equations (constraints) or qualitative type, expressed for example in the form of logical relationships. The methods for pattern recognition basic aim to identify an area of values that correspond to failed states. The methods based on Bayesian networks are probabilistic methods that allow making the diagnosis. The methods based on the tree decision can build a tree in successive issues. Depending on the response, this tree can be built and allows the diagnosis. The case-based methods include recording in a knowledge base effects observed defects that have occurred in the past and then, when acting occurs abnormal.

1) Terminology: As a step towards unified terminology, the Technical Committee of International Federation of Automatic Control "(IFAC) SAFEPROCESS suggested definitions in the field of fault diagnosis.

- Structural analysis: Analysis of the structural properties of models, i.e. properties that are independent of the actual values of the parameter.

- Failure: A permanent interruption of the ability of the system to perform a function required under specified operating conditions.

- Default: An unlicensed deviation of at least one structural property or characteristic parameter of the system in relation to the nominal behavior (usual or acceptable).

- Default detection: The determination of the presence of defects and the moment of their occurrences.

- Insulation of fault: The determination of the type, location and time of occurrence of a defect.

- Identification: The determination of the size and temporal behavior of a defect.

- Diagnostic: Determination of type, size, location and time of occurrence a defect; it tracks the detection of defects and includes isolation and identification.

- Failure effect: The consequence of a failure mode on the operation, function, or status of a variable.

- Qualitative model: A system model describing behavior with relationships between variables and system parameters in heuristic terms such as causalities or rules.

- Quantitative model: A system model describing behavior with relationships between variables and system parameters in analytic terms such as equations differences or differences.

- Modeling of the defect: Determination of a mathematical model to describe a specific effect of the defect.
- Re setup: Change of the structure and parameters of the controller. The original goal of Command is reached although execution may be degraded.

- Analytical redundancy: Determining a variable by measuring or using a mathematical model of the process under consideration.

- Residue: Signals carrying information, based on the difference between measurements and calculations based on the model.

- Threshold: The limit value of the deviation of a residual with the zero, so if it is exceeded, a defect is declared as detected.

2) Methods of diagnosis: The diagnostic strategy and the form in which knowledge is available to determine the methods used to design the monitoring algorithms. The main criterion for classification of monitoring methods is based on the type of knowledge; there are two types of approaches methods: the methods using operating models and those using diagnostic models (often known as the methods with or without model).

3) Methods without models: Described by several realization modes, methods without a model as the name implies, have no operating model. Otherwise, no model is describing normal behavior and delinquent the behavior of the system. The methods are then called learning processes and pattern recognition [2] or artificial intelligence. [3] The model without method is used in the case of complex systems dealing with very varied data (analog, digital) or imprecise (noise measurement). The goal of pattern recognition is to automatically classify shapes in modes (classes) known a priori. Therefore, these techniques must know a priori at all operating states (normal and default).

Analysis Phase: This phase involves the gathering of all existing information on the data process from the various sensors, models of devices, history, and experience. From this phase, we must get a precise definition of the parameters that will observe or represent the operating modes. A series of observations of the same model is not a single point but occupies an area of the observation space. This is due to the noise inherent in the various measures. It is therefore associated with each observed form of a mode or class. After representing the modes by significant parameters, we try to have as much data on each observable mode; this is the learning phase.

The phase of selection of a detection system: The boundaries between the classes are defined in this phase. A decision system capable to decide the membership or not of a new observation to existing classes is created. Many solutions used to define the decision system can be retained. [2] Cites a non-exhaustive methods of parametric discrimination with rejection (assume full knowledge of probability laws governing observations and applied under the Gaussian assumption with parameter estimation), methods of nonparametric discrimination (laws probability in each class are unknown but can be estimated by the method of Parzen or 
the nearest neighbor), Operating phase. It is the implementation phase of the diagnostic system to propose a decision for any new observation made. The system must adapt its decision rule based on new classes detected.

4) Methods this second family of models: (Which is also our study) is based on the existence of material or analytical redundancy to characterize the operation mode or the system status. The principle of redundancy is to determine the value of variables in different ways and to analyze whether the results coincide. Physical redundancy consists of using multiple sensors for measuring the same variable. These sensors indicate the normal operation of the same value of noise and inaccuracies of close measurement. The duplication of the sensors is necessary to detect single faults. However, the location of the failure requires a minimum of three sensors, we will proceed to a majority vote. This method has been widely used in industry because it is easy to apply and very reliable, however, it is very heavy and expensive. Furthermore, it is not always applicable for architectural and material reasons and its scope is limited to only sensors failures. Unlike hardware redundancy, analytical redundancy allows finding the relationships between the known variables of the system (inputs and outputs). Many works are devoted to them, including the summaries found in [4], [5], [6] and the teaching manual [7] for linear systems. In this case, the generation of waste can be achieved by different approaches:

5) Approach parameter estimation: The input and output signal systems are used to estimate the different values of the analytical model parameters. The system parameters are estimated using identification procedures. The differences between the estimated values and the reference values are the residues. When the nominal values are not known, another approach is consisting to replace them with earlier estimates. In this case, the gap residues concerning zero are the result of parameter variations. These differences are then analyzed by the decision theory [4], [8].

The approach of state estimation: This is the dual approach to the estimation of parameters. The state estimation techniques use the raw database to estimate the state of the system $x(t)$ and consequently its output $y(t)$. These are compared with the actual outputs: any deviation between the measured value of the output and the actual value is a residue. There are two classes of approaches, the first one estimates the state vector (Luenberger observer [9] Kalman filter [10] and deduces the estimated output by applying the measurement equation. the other approach uses the observers who feel directly outputs, or more generally any combination of the state variables including the theoretical behavior is known in normal operation. [11].

The approach of parity space: This approach led to a rewriting of the equations of state and measurement, in which only known variables (commands and outputs) are allowed to appear. In the linear case, these equations are known by the parity equations and analytical redundancy relations (RRA) in the most general case.

\section{RoBUSt DiAgNOSIS BY BOND GRAPH APPROACH}

The diagnosis systems by Bond Graph approach is one of the methods that are based on the use of a mathematical model of the system. Analytical methods rely on a knowledge of the system established by the explicit formulation of an analytical model of the monitored system. The basic principle of this approach is based on the acquisition of information through sensors on the monitoring process. The comparison between the actual behavior of the process and the behavior predicted by the model provides the information in a set of signals indicative of defects (residues). Analysis (time or frequency) of these signals can detect and optionally interpret any abnormal behavior of the system and locate its origin. From a representation by a bond graph approach, a monitoring system can be illustrated in Fig. 1. There are essentially two parts: one for the transfer of power and energy (formed by the process and all of the actuators), while the second shows the signals (information system is the sensors and the control system).

The bond graph model represents the energy of the system. The process is generally modeled by the usual elements of bond graphs (R, C, I, and the junctions). The actuators (pump, heat source,) are modeled by the sources (stress and/or flow). The sources can be simple (Se, Sf) or modulated (MSe, MSf ), which is controlled by an external signal provided by a controller or operator. The sensors and the control system form the information system. In the first system (energy), the exchanged power is represented by a half-arrow (a power link) led the effort and the flow variables. In the second system (information system) the exchanged power is negligible, it is then represented by an information link (arrow) which is the same used in the classic block diagrams.

Monitoring algorithms (IDF detection and fault isolation) receive online information from the sensors (sensors of effort and flow) and withdraw the supervision system alarms. The information on the status of faulty elements is transmitted to the maintenance service. In the following sections, the different approaches to a bond graph by IDF are presented. There are two main approaches to bond graph process monitoring: the quantitative approach and the qualitative approach. Monitoring algorithms (IDF detection and fault isolation) receive online information from the sensors (sensors of effort and flow) and withdraw the supervision system alarms.

1) The qualitative approach for diagnosis using the bond graph: This approach does not require a very precise model. Unlike conventional representations of knowledge that describe the structure of the system and its status through various tools (block diagram, differential equations, etc.), the qualitative leap graphs explicitly describe the location of the system components and their interconnections. Thereafter, the based surveillance modeling qualitative bond graph is simpler in comparison with quantitative methods because it does not require a specific model. These qualitative models are built without consideration of system parameters. They are based on qualitative values (instead of numerical values) defined by the set $\{[1][+][0][-][-1][?]\}$ Representing the quality of the deviation in the space measures compared to the normal 
operation. Operators are thus qualitative, they can be defined using the operators of real numbers $\{+,-, \mathrm{x}, \div,=\}[12]$.

We then solve a system of qualitative equations to determine the cause of failures. Other studies use temporal causal graphs to isolate failures taking into account the dynamics (and the order) of occurrence of the fault. [12] Other authors use qualitative analysis of linear equations of state to determine possible causes of failure. [13] The advantage of this approach is that it does not require a deep knowledge of the system structure or numerical magnitudes of the parameters. However, this approach becomes a complicated process for multi-energy and has other drawbacks such as non-fault detection sensors and the difficulty of determining the lower and upper limits of the deviations [14].

2) The quantitative approach for diagnosis using the bond graph: The principle of this approach is to compare the normal behavior of the process with the digital model. Unlike the qualitative approach, the quantitative approach is based on physical laws and requires a deep knowledge of the system structure and the numerical values of the parameters. Models whatever their forms (transfer function, equations of state, ) are obtained based on physical laws (first principles laws) [15] or the basis of a statistical parameter identification [16], [5]. In the remainder of this section, we will outline the methods of diagnosis based models using the Bond Graph approach.

3) Observers to enter unknown: An observer is defined as an unknown input because the estimation error of the state vector tends to zero asymptotically without taking into account the unknown input (for example, a disturbance) in the system [17]. The following LTI system:

$$
\begin{aligned}
& x(t)=A x(t)+B u(t)+ \\
& D v(t), y(t)=C x(t)
\end{aligned}
$$

$$
\text { Where } x(t) \in R n_{\text {is the state vector, }} u(t) \in R m \text { is }
$$
the measurable input vector, $v(t) \in R q$ of the unknown input vector, $y(t) \in R p$ is the output vector. The observer unknown input of this system is:

$$
\begin{aligned}
& z(t)=N z(t)+L y(t)+G u(t), \\
& x 0(t)=z(t)-E y(t)
\end{aligned}
$$

Where $z(t) \in R n$ is the state vector, the matrices $N, L, G E$ and have suitable dimensions and are determined such that $x \mathrm{O}(t)$ converges asymptotically to $x(t)$ the dynamics of the error is given by:

$$
\begin{aligned}
& e(t)=x(t)-x 0(t) e(t)=x(t)-X 0(t)= \\
& A x(t)+B u(t)+D v(t)-Z(t)+e y(t)= \\
& A x(t)+B u(t)+D v(t)-N z(t)-L y(t)- \\
& G u(t)+E C(A x(t)+B u(t)+D v(t))=
\end{aligned}
$$

$$
\begin{aligned}
& A x(t)+B u(t)+D v(t)-n x 0(t)+N e y(t)-L y(t) \\
& -G u(t)+E C(A x(t)+B u(t)+D v(t))= \\
& N x(t)-n x 0(t)-(N P+L C-P A) x(t) \\
& -(G-P B) u(t)+P D v(t)= \\
& N e(t)-(L C+N P-P A) x(t)- \\
& (G-P B) u(t)+P D v(t)
\end{aligned}
$$

Where $P=I+E C I \in R n * n$ and unit matrix. The error converges to zero if:

$$
\begin{aligned}
& P D=D+E D C=0 \\
& N P+L C-P A=0 \\
& G-P B=0
\end{aligned}
$$

The solution of equation (66) for E can be written as:

$E=D(C D)+Y(I p-(C D)(C D)+)$

Wherein $(C D)+$

is the generalized inverse of $C D, I p \in R p * p$ the unit, $Y$ is an arbitrary matrix of appropriate size. The $G$ matrix may be derived from equation (57) as follows:

$G=P B=B+E C B$

The state error converges to zero as the poles $N$ are stable. Let $K$ us:

$N=P A-K C$

The equation is true iff :

$K=L+D O$

$N=P A-K C=P A-(L+N) C=$

$P A-L C-N E C N E C$

$+\Rightarrow N=P A-L C$ or $N(I+E C)=$

$N P=P A-L C$

And as $\mathrm{P}$ is known, the poles $\mathrm{N}$ can be placed carefully choosing the matrix K (eq. (11)).

The closed-loop gains of the observer are:

$$
\begin{aligned}
& L=K-K=N E-(P A-K C) E= \\
& K(I+E C)-P A E
\end{aligned}
$$

Calculation of matrix $\mathrm{N}, \mathrm{G}$, and $\mathrm{L}$ complete the construction of the observer unknown complete order entry. To isolate the fault, the disturbances must be redefined 52 equation system as follows: 
$x(t)=A x(t)+B u(t)+E D D(t)+$

Ef $f(t), y(t)=C x(t)+$

Gdd $(t) G f+f(t)$

where $\mathrm{x}(\mathrm{t})$ is the state vector, $\mathrm{y}(\mathrm{t})$ is the output vector, $\mathrm{u}$ $(t)$ is the known input vector, $d(t)$ is the unknown input vector (disturbance), $\mathrm{f}(\mathrm{t})$ is the defects vector, the matrices $A, B, C, D, E d, E f, G d$ and $G f$ are constant. An observer unknown input with its waste generator is defined by:

$$
\begin{aligned}
& x 0(t)=H x x 0(t)+H u u(t)+H y y(t), \\
& r(t)=\operatorname{Mxx} 0(t)+\operatorname{Myy}(t)
\end{aligned}
$$

Where $\boldsymbol{H} \boldsymbol{x} \boldsymbol{H u}, \boldsymbol{H y}, \boldsymbol{M x} \boldsymbol{M} \boldsymbol{y}$ and have appropriate dimensions. Either the error defined by $e(t)=x 0(t)-T x(t)$, where $T$ is a transformation matrix. The estimation error can be written as follows:

$$
\begin{aligned}
& E(t)=X 0(t)-T x(t)= \\
& H x x 0(t)+H u и(t)+H y y(t) \\
& -T A x(t)-t B u(t)- \\
& T E D D(t)-T E f f(t)= \\
& H x x 0(t)-H X T x(t)+H X T x(t) \\
& +H u u(t)+H y(C(t)+G d d(t)+G f f(t))- \\
& T A x(t)-t B u(t)-T E D D(t)-T E f f(t)= \\
& H x(x 0(t)-T x(t))+(H X T+H y C-T A) x(t) \\
& +(H u-T B) u(t)+(H y G d-T E d) d(t)+ \\
& (H y G f-T E f) f(t)= \\
& H X E(t)+(H X T+H y C-T A) x(t) \\
& +(H u-T B) u(t)+ \\
& (H y G d-T E d) d(t)+(H y G f-T E f) f(t) .
\end{aligned}
$$

The residue vector is given by:

$$
\begin{aligned}
& r(t)=M_{X} E(t)+(M y C-M x T) x(t) \\
& +M y G d d(t)+M y G f f(t)
\end{aligned}
$$

The asymptotic convergence of the error and the decoupling of the disturbances are verified with the following conditions: $H x$ is stable,

$$
\begin{aligned}
& T A=-(H X T+H y C), \\
& T B=H u, H y G d-T E d \\
& =0, M y C-M x T=0,
\end{aligned}
$$$$
M y G d=0 \text {, }
$$

The estimation error and the residues are defined by:

$$
\begin{aligned}
E(t) & =H X E(t)+(H y G f-T E f) f(t), \\
r(t) & =\operatorname{MXE}(t)+\operatorname{MyGf} f(t)
\end{aligned}
$$

And as $H x$ is stable in healthy cases (without defects) $e(t) \rightarrow 0$ and $r(t) \rightarrow 0$ as $t \rightarrow \infty$.

a) Application with the Bond Graph approach: At this stage, we consider the system treated in section (Fig. 2).

The model in the state space is given by:

$\left\{\begin{array}{l}\dot{Q}_{2} \\ \dot{Q}_{7}\end{array}\right\}=\left[\begin{array}{cc}-\frac{1}{C_{T 1} R_{v b}} & \frac{1}{C_{T 2} R_{v b}} \\ \frac{1}{C 1 R_{v b}} & -\frac{1}{C_{T 2} R_{v b}}-\frac{1}{C_{T 2} R_{v o}}\end{array}\right]\left\{\begin{array}{l}Q_{2} \\ Q_{7}\end{array}\right\}+\left[\begin{array}{l}1 \\ 0\end{array}\right] Q_{P}$

Consider the elements $C: C_{T 1}, R: R_{v b} C: C_{T 2}$ and $R: R_{v o}$; candidate defects, which are reflected by leaks in tanks and leakage/blockage in the valve. If the fault is in the R-element: $\boldsymbol{R}_{v b}$ while Fig. 2(b) shows that this component gives the flow to the system. This element can be therefore replaced by a stream source (Fig. 3(a)). Thereafter we proceed to the construction of an observer unknown entry (taking into account the new source of flow disturbance like), then the residue takes a zero value if and only if the element $R: R_{v b}$ is in default. By following the same steps, one can construct the observer for the other combinations of defects (Fig. 3(a), (b) and (c)).

For Fig. 3(a) model in the state space is:

$$
\left\{\begin{array}{l}
\dot{Q}_{2} \\
\dot{Q}_{7}
\end{array}\right\}=\left[\begin{array}{cc}
0 & 0 \\
0 & -\frac{1}{C_{T 2} R_{v o}}
\end{array}\right]\left\{\begin{array}{l}
Q_{2} \\
Q_{7}
\end{array}\right\}+\left[\begin{array}{l}
1 \\
0
\end{array}\right] Q_{P}+\left[\begin{array}{c}
-1 \\
1
\end{array}\right] V(t)
$$

For the model in Fig. 3(b):

$$
\left\{\begin{array}{c}
\dot{Q}_{2} \\
\dot{Q}_{7}
\end{array}\right\}=\left[\begin{array}{cc}
-\frac{1}{C_{T 1} R_{v b}} & 0 \\
\frac{1}{C 1 R b} & 0
\end{array}\right]\left\{\begin{array}{l}
Q_{2} \\
Q_{7}
\end{array}\right\}+\left[\begin{array}{l}
1 \\
0
\end{array}\right] Q_{P}+\left[\begin{array}{c}
-\frac{1}{R_{v b}} \\
-\frac{1}{R_{v b}}
\end{array}\right] V(t)
$$

Finally, Fig. 3@ gives:

$$
\left\{\begin{array}{c}
\dot{Q}_{2} \\
\dot{Q}_{7}
\end{array}\right\}=\left[\begin{array}{cc}
-\frac{1}{C_{T 1} R_{v b}} & \frac{1}{C_{T 2} R_{v b}} \\
\frac{1}{C 1 R_{v b}} & -\frac{1}{C_{T 2} R_{v b}}
\end{array}\right]\left\{\begin{array}{l}
Q_{2} \\
Q_{7}
\end{array}\right\}+\left[\begin{array}{l}
1 \\
0
\end{array}\right] Q_{P}+\left[\begin{array}{c}
0 \\
-1
\end{array}\right] V(t)
$$

We note that the matrix $\mathrm{A}$ in equation (16) is different from those found in equations (15), (16), (17), in each case, we must build a corresponding observer. In the case of a fault in the element R: RVO, 14 the equation can be rewritten as follows:

$$
\left\{\begin{array}{l}
\dot{Q}_{2} \\
\dot{Q}_{7}
\end{array}\right\}=\left[\begin{array}{cc}
-\frac{1}{C_{T 1} R_{v b}} & \frac{1}{C_{T 2} R_{v b}} \\
\frac{1}{C 1 R_{v b}} & -\frac{1}{C_{T 2} R_{v b}}-\frac{1}{C_{T 2} R_{v o}+\Delta R_{v o}}
\end{array}\right]\left\{\begin{array}{l}
Q_{2} \\
Q_{7}
\end{array}\right\}+\left[\begin{array}{l}
1 \\
0
\end{array}\right] Q_{P}
$$

$$
\begin{aligned}
& \text { Or } \\
& x(t)=A x(t)+B u(t)+\Delta A x(t) \\
& =A x(t)+B u(t)+D v(t)
\end{aligned}
$$




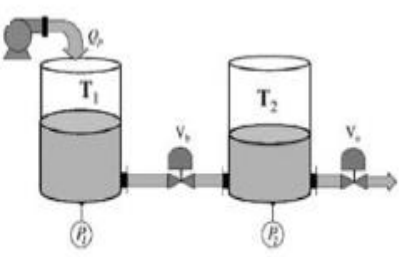

(a)

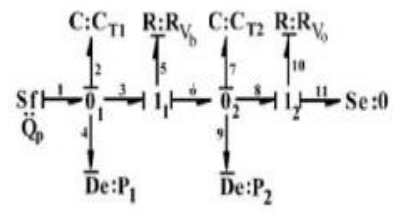

(b)
Fig. 2. System 2 Tanks (a) Bond Graph Equivalent (b).

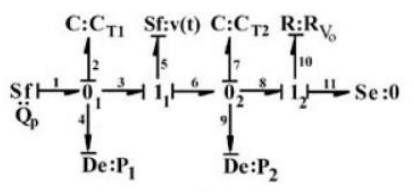

(a)

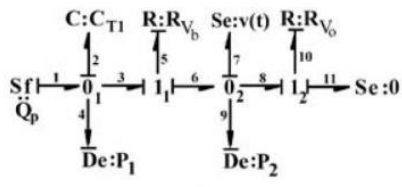

(b)

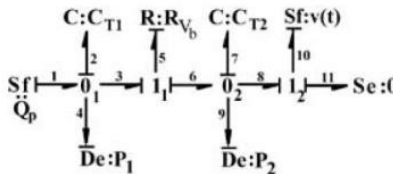

(c)
Fig. 3. Different Cases of unknown Input Assumptions Considered for Defects.

Where, dA represented the change in matrix $\mathrm{A}$ due to a parametric failure, its effect results in an unknown input. The formulation presented in equation (23) can be implemented in the model Bond Graph by the appropriate addition of stress sources and flows. The model Bond Graph modified to a defect in R: Rgb and C: CT2 respectively are shown in Fig. 4(a) and (b).

b) Discussions: The representation of the unknown inputs in a bond graph model does not generalize the method to design observers to unknown entries in a direct way (from the bond graph model). Construction observers must necessarily go through the step of writing the state equations from the model. Under these conditions, the use of the Bond Graph approach is not practical. In the remainder of this article, we will introduce other approaches for diagnosis based on the bond graph model.

4) Residues in the parity area outside: Consider the following LTI system:

$$
\begin{aligned}
& \dot{X}(t)=A x(t)+B u(t)+E_{d} d(t)+E_{f} f(t) \\
& Y(t)=C x(t)+D u(t)+G_{d} d(t)+G_{f} f(t)
\end{aligned}
$$

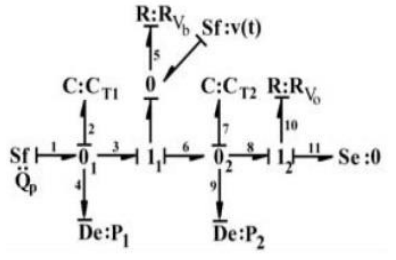

(a)

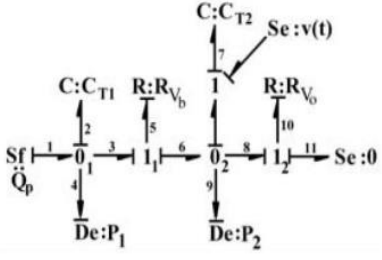

(b)
Fig. 4. Bond Graph with unknown Grafted.
In normal operation $\mathrm{f}(\mathrm{t})=0 \forall \mathrm{t}$. It is assumed initially that $(\mathrm{d}(\mathrm{t})=0, \mathrm{f}(\mathrm{t})=0)$.

$$
\begin{aligned}
& Y(t)=C x(t)+D u(t) \\
& \dot{Y}(t)=C \dot{x}(t)+D \dot{u}(t)
\end{aligned}
$$

Substituting $\mathrm{x}=\mathrm{Ax}(\mathrm{t})+\mathrm{Bu}(\mathrm{t})$ in equation (27) and deriving up to the order $\mathrm{m}$ :

$$
\begin{aligned}
& \dot{Y}(t)=C A x(t)+C B u(t)+D \dot{u}(t) \\
& \ddot{Y}(t)=C A^{2} x(t)+C A B u(t)+C B \dot{u}(t)+D \ddot{u}(t) \\
& \vdots \\
& Y^{m}(t)=C A^{m} x(t)+C A^{m-1} B u(t)+\ldots+C B u^{m-1}(t)+D u^{m}(t)
\end{aligned}
$$

If the matrix is full rank, then you can apply the theorem Caley Hamilton (Each matrix satisfies its characteristic equation that is to say):

$$
\phi(A)=0
$$

Such that:

$$
\phi(s)=|s I-A|=s n+a n-1 s n-1+\ldots+a 0+A 1 S)
$$

Then:

$$
\begin{aligned}
& Y^{n}(t)=C A^{n} x(t)+C A^{n-1} B u(t)+\ldots+C B u^{n-1}(t)+D u^{n}(t) \\
& =a_{n-1} C A^{n-1} x(t)-a_{n-2} C A^{n-2} x(t)-\ldots-a_{1} C A x(t) \\
& -a_{0} C x(t)+C A^{n-1} B u(t)+\ldots+C B u^{n-1}(t)+D u^{n}(t) \\
& =-a_{n-1}\left(y^{n-1}(t)-C A^{n-2} B u(t)-\ldots-C B u^{n-2}-D u^{n-1}(t)\right. \\
& -a_{n-2}\left(y^{n-2}(t)-C A^{n-3} B u(t)-\ldots-C B u^{n-3}(t)-D u^{n-1}(t)\right. \\
& -\ldots \\
& -a_{0}(y(t)-D u(t)) \\
& +C A^{n-1} B u(t)+\ldots+C B u^{n-1}(t)+D u^{n}(t)
\end{aligned}
$$

Or:

$\phi_{A R R}\left((y(t), u(t)),(\dot{y}(t), \dot{u}(t)), \ldots,\left(y^{n}(t), u^{n}(t)\right)\right)=0$

Equation (27) defines a set of constraints between the inputs/outputs, which are by definition the Analytical Redundancy Relations.

a) Sample Application: Consider the mass-springdamper system and its bond graph model represented by Fig. 5(a) and (b).

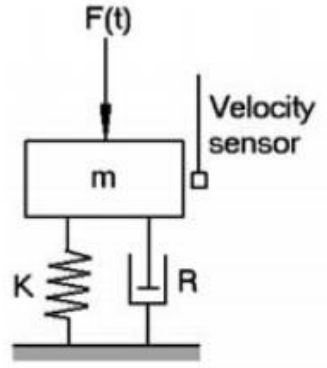

(a)

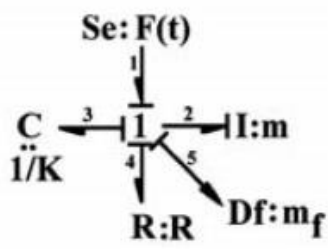

(b)
Fig. 5. Bond Graph Model of a System Mass-Spring-Damper. 
From the link graph model, the equations of the state space are as follows:

$$
\begin{aligned}
& \left\{\begin{array}{l}
\dot{x}_{1} \\
\dot{x}_{2}
\end{array}\right\}=\left[\begin{array}{cc}
-\frac{R}{m} & -K \\
\frac{1}{m} & 0
\end{array}\right]\left\{\begin{array}{l}
x_{1} \\
x_{2}
\end{array}\right\}+\left[\begin{array}{l}
1 \\
0
\end{array}\right] u(t) \\
& \left\{y_{1}\right\}=\left[\begin{array}{ll}
\frac{1}{m} & 0
\end{array}\right]\left\{\begin{array}{l}
x_{1} \\
x_{2}
\end{array}\right\}
\end{aligned}
$$

Where $x 1=P 2, x 2=Q 3, u(t)=F(t)$ and $\mathrm{y} 1$ is the output of the speed sensor. By applying several derived from $\mathrm{y} 1$ :

$$
\begin{aligned}
& \dot{y}_{1}=\frac{\dot{x}_{1}}{m}=-\left(\frac{R}{m^{2}}\right) x_{1}-\left(\frac{K}{m}\right) x_{2}+\frac{u(t)}{m} \\
& =-\left(\frac{R}{m}\right) y_{1}-\left(\frac{K}{m}\right) x_{2}+\frac{u(t)}{m}, \\
& \ddot{y}_{1}=-\left(\frac{R}{m}\right) \dot{y}_{1}-\left(\frac{K}{m}\right) \dot{x}_{2}+\frac{\dot{u}(t)}{m} \\
& =-\left(\frac{R}{m}\right) \dot{y}_{1}-\left(\frac{K}{m^{2}}\right) x_{1}+\frac{\dot{u}(t)}{m}
\end{aligned}
$$

We thus obtain an RRA (Relationship Analytical Redundancy)

$$
m \ddot{y}_{1}+R \dot{y}_{1}+K y_{1}-\dot{u}(t)=0
$$

If we add a vertical displacement sensor (y2):

$$
\left\{\begin{array}{l}
y_{1} \\
y_{2}
\end{array}\right\}=\left[\begin{array}{cc}
\frac{1}{m} & 0 \\
0 & 1
\end{array}\right]\left\{\begin{array}{l}
x_{1} \\
x_{2}
\end{array}\right\}
$$

We then get the following RRA:

$$
\begin{aligned}
& m \dot{y}_{1}+R y_{1}+K y_{2}-u(t)=0, \\
& y_{1}-\dot{y}_{2}=0
\end{aligned}
$$

As a conclusion, we can note from the previous example that, the maximum degree spin in the RRA is "1". The first analytical redundancy relationship RRA1 is responsive to a static shift concerning the valuesy1 and y2, the second RRA2 is responsive to a static lag y1. If RRA is written with a higher-order derivative, the detection of the shift in the sensors will not be possible. Superior derived from a noise signal will also cause problems in the decision step.

\section{5) Analytical redundancy relations generation:}

a) Graphical representation systems: Before presenting the algorithm for generating the RRAS, we first wanted to highlight some graphical representations of dynamical systems. For this, we have chosen to proceed with a simple example shown in Fig. 6.

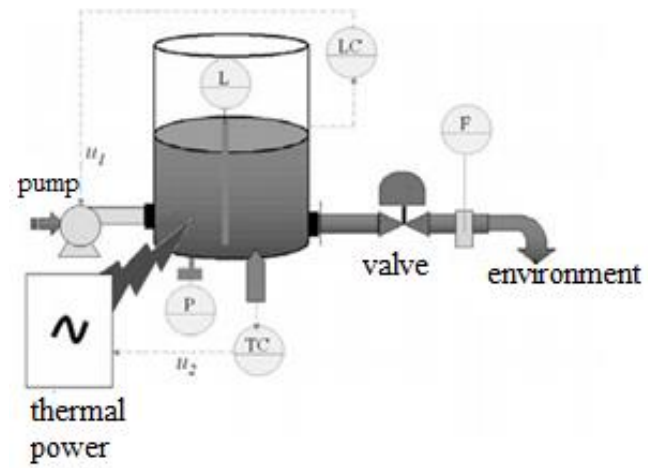

Fig. 6. Tank Supplied with Water by a Pump.

b) Representation by a structural model: It is shown in [18] that, from a structural standpoint, the dynamic model of any physical system may be represented by the pair [C, Z], with $\mathrm{Z}=\{\mathrm{z} 1, \mathrm{z} 2 \ldots \mathrm{zn}\}$ represents the set variables and the system parameters, and $\mathrm{C}=\{\mathrm{c} 1, \mathrm{c} 2, \mathrm{cn}\}$ represents the set of constraints. Then, the structural representation will express the relationship between the variables and the constraints of the process. The advantage of this representation is that it can represent any system regardless of its model (qualitative, quantitative, static, dynamic, rules, tables, etc.). Thus, the analytical form of the system model is not necessarily required. By applying the description given in [18], the constraints corresponding to the hydraulic part of the system of Fig. 6 are given in Table I. Where M (t) is the total mass in the tank, qi (t) is the input flow, qo (t) is the output rate, $\mathrm{u}(\mathrm{t})$ is the control of the level controller, the coefficient $\mathrm{Cd}$ discharge valve, and the A section of the tank and ФPi the function corresponding to the level controller. In addition to these relationships, an eighth constraint can be added by introducing the derivative of the variable $M(t)$ : qo ( $t$ ) output rate, $\mathrm{u}(\mathrm{t})$ the control of the level controller, the discharge coefficient $\mathrm{Cd}$ of the valve, the section A of the tank and $\Phi \mathrm{Pi}$ the function corresponding to the level controller. In addition to these relationships, an eighth constraint can be added by introducing the derivative of the variable $M(t)$ :

TABLE. I. Structural Model of THE Hydraulic Part

\begin{tabular}{|l|l|l|}
\hline Component & Constraint & Model \\
\hline Tank & $C_{1}$ & $M(t)=q_{i}(t)-q_{0}(t)$ \\
\hline Pump & $C_{2}$ & $q_{i}(t)=\alpha u(t)$ \\
\hline Valve & $C_{3}$ & $q_{0}(t)=C_{d} \cdot \sqrt{M(t)}$ \\
\hline level sensor & $C_{4}$ & $L(t)=\frac{M(t)}{\rho A}$ \\
\hline Pressure sensor & $C_{5}$ & $P(t)=M(t) \cdot \frac{\rho}{A}$ \\
\hline flow sensor & $C_{6}$ & $F(t)=q_{0}(t)$ \\
\hline level regulator & $C_{7}$ & $u(t)=\phi_{P I}(M(t))$ \\
\hline
\end{tabular}


$c_{8}: \dot{M}(t)=\frac{d M(t)}{d t}$

The set of constraints $\mathrm{C}$ and all $\mathrm{Z}$ variables of the physical system are therefore determined as follows:

$C=\left\{c_{1}, c_{2}, c_{3}, c_{4}, c_{5}, c_{6}, c_{7}, c_{8}\right\}$

$Z=\left\{M(t), M(t), q_{i}(t), q_{0}(t), u(t), L(t), P(t), F(t)\right\}$

Note that the structural model is used to determine the structural properties of the systems (observability, controllability, surveillabilité, and reconfigurability) independently of numerical values of the parameters.

Representation by a digraph

Consider the model of the example in Fig. 6 in the form of the following state equations:

$$
\begin{aligned}
& \dot{x}(t) f(x(t), u(t))=-C_{d} \cdot \sqrt{x(t)}+\alpha \cdot u(t), \\
& y(t)=g(x(t), u(t))=\left(\begin{array}{l}
y_{1}(t) \\
y_{2}(t) \\
y_{3}(t)
\end{array}\right)=\left(\begin{array}{c}
\frac{1}{\rho^{A}} \cdot x(t) \\
\frac{2}{A} \cdot x(t) \\
C_{d} \cdot \sqrt{x(t)}
\end{array}\right)
\end{aligned}
$$

The digraph corresponding to the system of equation (35) is a graph in which the vertices are the inputs, the outputs and the states of the system; and the links between the vertices are defined by the following rules:

- There exists a link xk top (respectively top ul) to the vertex $\mathrm{xi}$ if and only if the state variable xk (respectively ul input variable) appears in the function fi.

- There is a link to the top xk yi higher if and only if the state variable xk appears in the function gj. Fig. 7.

The digraph associated with equation (35) is given in

The representation by the digraph is an abstraction of the dynamic model of the moment that the tops can be interpreted as a mutual influence between the variables of the system.

The disadvantage of this form of representation is that it does not represent the algebraic constraints of the model.

Representation by bond graph: The bond graph model, derived causality, corresponding to the hydraulic part of the physical system of Fig. 6 is shown in Fig. 8.

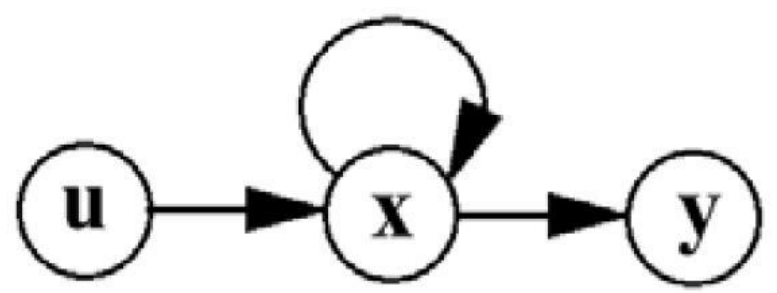

Fig. 7. A Logging the Equations Corresponding to 35.

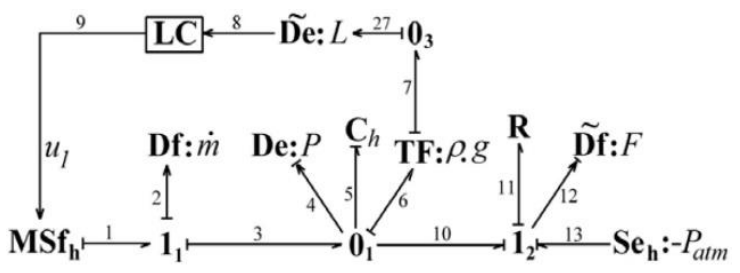

Fig. 8. Bond Graph Model of the Example in Fig. 6.

Using the bond graph representation, the set of variables $\mathrm{Z}$ (known and unknown $\mathrm{K} \mathrm{X}$ ) and the set of constraints $\mathrm{C}$ can be deducted directly from the bond graph model of the process.

c) Generation RRAS using the bond graph: The purpose of using the graphical representation of links is to use a single tool for modeling, the RRAS generation, the structural analysis, and the monitoring and sensor placement. A bond graph monitoring system can be represented by the diagram shown in Fig. 1. There are essentially two parts: the bond graph model and the information system. The bond graph model represents the energy of the physical process. It includes the process and all the actuators which are modeled by the sources (effort or flow). The sources may be single: Se, Sf (pump, heating, supply pressure, etc.) or modulated MSe, MSf (sources controlled by an external signal provided by a controller or a user). The sensors and the control system (PID, $\mathrm{T} R$, etc.) form the information system. In the first system (energy), the power exchanged is represented by a half-arrow (link) that translates the power of variables (effort and flow). In the second system (information system) the power exchanged is negligible; it is represented by an information link.

The generation of RRAS through the bond graph methodology is based on the same principles as in the bipartite graph, namely the elimination of unknowns in an overdetermined and observable subsystem. On a bond graph model, the observability can be checked using the structural properties developed in [19]. The known variables $\mathrm{K}$ are those of the detectors and the sources while the unknown variables $\mathrm{X}$ are those of the links of powers in the elements $\mathrm{C}, \mathrm{I}$ and $\mathrm{R}$. The elimination of unknown variables is routinely on the bond graph model with a causal property and a course of causal paths. In the following, the developed approach consists of generating an algorithmic and systematic way of the RRAS corresponding to the residues and the failure's signature matrix. In a bond graph representation, the relationship on $f(K)=0$ the definition of an RRA becomes:

$$
f\left(D_{e}, D_{f}, S_{e}, S_{f}, M S e, M S f, \theta_{m}\right)=0
$$

With .theta..sub.m represents all the measured or estimated system parameters. The algorithm presented here allows generating all the possible RRAS from a bond graph model of a given system. The model is first set preferably on the derived causality (with inversion of the detectors if possible). This avoids the initial conditions of the state and considers the sources and detectors as inputs of the model. The RRAS, in differential form, is then directly deduced from the expressions of the laws of junction 0 and 1 of the leap in the model graph causally derived. 
RRAS generation algorithm by bond graph: The following algorithm can generate RRAS systematically from a bond graph model. Here, in order, the steps to follow:

1) Put the bond graph model causality preferably derived (by reversing the causal sensors if possible).

2) Write the equations of the resulting model: behavior FB, Junctions FJ to measured FY, sources FAT and control FC.

3) For any junction equation 0 and 1 containing at least one detector:

a) eliminate unknown variables by browsing the causal paths on the bond graph,

b) for any detector whose causality is reversed an RRA is deducted,

c) for any detector whose causality cannot be reversed an RRA is deduced by making equal its output with the output of another sensor of the same type (hardware redundancy).

4) RRA is obtained from each controller by comparing the measured output with output predicted by the control algorithm.

5) Repeat steps 3 and 4:

a) if the RRAS obtained are strictly different from those already obtained then keep, otherwise continue until all equations junctions and those Regulators are explored. Now apply this algorithm on our example application given to Fig. 3.

6) The bond graph model causality derivative corresponding to the hydraulic part the system is given to Fig. 8. The different analytical constraints model is given by equations (25), (27), (29), (31) and (32). Moreover, it is Important, in this algorithm, to consider that the outputs of sources and regulators are known (measured). Step 3 the algorithm is to eliminate the unknown variables of each equation junction. Choose as the first equation corresponding to junction 01 .

So we have:

$$
f_{6}=f_{3}-f_{4}-f_{5}-f_{10}
$$

In this equation, the subset of unknown variables is $\mathrm{X}=$ f6, f3, f4, f5, f10. These variables can be eliminated by browsing the causal paths on the bond graph model in Fig. 8. $\mathrm{f} 4$ and $\mathrm{f} 6$ correspond to variable flow pressure sensors by $\mathrm{P}$ and level $\mathrm{L}$ and therefore their values are zero $(\mathrm{f} 6=\mathrm{f} 4=0)$. $\mathrm{f3}$ can be determined from the causal path MSfh: $\Phi$ pump (U1) $\rightarrow$ $\rightarrow \mathrm{fl}$ f3 (see Fig. 8), since the output of the actuator is considered in this case as being known (MSf pump $=\Phi(\mathrm{u} 1))$. We can write:

$f_{3}=\phi_{\text {pump }}\left(\boldsymbol{u}_{1}\right)$

The causal path Df: $\mathrm{F} \rightarrow \rightarrow \mathrm{fl} 2$ f10 f10 determines the variable:
$f_{10}=F$

While f5 variable is calculated from the constitutive relationship of the element $C_{h}$ :

$f_{5}=C_{h} \frac{d e_{5}}{d x}$

Where $\mathrm{Ch}$ is given and $\mathrm{e} 5$ is determined by traversing the causal path from:

$e 27 L \rightarrow \rightarrow \rightarrow e 7 e 6 \rightarrow e 5 e 5=\Rightarrow \rho . g L$

Was thus:

$f_{5}=\frac{A}{g} \rho g \frac{d L}{d t}=A \rho \dot{L}$

All unknown variables in equation (40) are eliminated, the first following analytical redundancy relationship is then obtained:

$R R A_{1}: \phi_{\text {pump }}\left(u_{1}\right)-\rho A \dot{L}-F=0$

Proceeding in the same way as in equation (39), a second RRA may be generated from the equation of junction 01 . In this equation, e12 = 0 since it corresponds to the variable force of a flow detector (Df: F). We can write:

$e_{12}=e_{10}-e_{11}+e_{13} \Rightarrow e_{10}-e_{11}+e_{13}=0$

From the causal path:

$e_{27} L \rightarrow \rightarrow \rightarrow e_{7} e_{6} \rightarrow e_{10}$

$e_{10}$ calculates $e_{13}$ Variable represents the atmospheric pressure of the ambient environment, while $e_{11}$ can be obtained from the constitutive relation of the element $\mathrm{R}$ :

$e_{10}=\rho g L$

$e_{13}=-P_{a t m}$

$e_{11}=\phi_{R}\left(f_{11}\right)=\phi_{R}(F)$ first is

The second RRA which is structurally independent of the $R R A_{2}=\rho g L-\phi_{R}(F)-P_{a t m}=0$

The Prior RRAS (Eq. (39) and Eq. (41)) are both obtained from the junction corresponding to the hydraulic phenomena of the process.

Failures signature Matrix: The RRAS form the structure of a binary matrix $\mathrm{Sji}$ which provides the information on the sensitivity of the residues to the failures component of the physical process (sensors, actuators, controllers, physical elements). The matrix elements are defined as follows:

$S_{j i}=\left\{\begin{array}{c}S_{j i}=1, \text { if the } i^{\text {th }} \text { RRA contains variables of } j^{\text {th }} \text { component } \\ 0, \text { else }\end{array}\right.$ 
The Sji matrix is called a failure signature matrix that provides the logic for the location errors detected during the system operation. The purpose of the localization (isolation) procedure is to provide the operator with a list of failed components. Each component has a signature represented by a vector line of the matrix and does not take into account the values assigned to the variables $\mathrm{Db}$ (detectability) and $\mathrm{Ib}$ (isolability). Component failure is detected if the variable associated with the component is present in at least one RRA (or the corresponding $\mathrm{r}$ residue). This failure is localizable if its signature is unique, that is to say, different from the signatures of the other components. From the specifications requested, we can fix the list of components to be monitored. Obviously, the performance of the monitoring system (about isolabilité) depends on the number of items to be monitored and to the differences in their failure signatures. To build the matrix of the signatures, some components are removed from the matrix since they are considered infallible.

How detection and fault isolation? Once the failures generated by the analytic redundancy relationships and the signature matrix have been obtained, the decision process is the next step in designing a monitoring system. It consists of making the detection and location of faults robust, reliable and efficient. In general, the logic used to determine whether or not a failure has occurred depends on the type of knowledge available about the process, the method used and treated as failures. A wide range of decision algorithms is given in [20].

The decision procedure is applied to all $\mathrm{R}$ residues $r-f(K)=0$ to generate a binary coherent vector $\mathrm{V}=[\mathrm{v} 1$ $\mathrm{v} 2 \ldots \mathrm{vn}$ ], which indicates whether a failure is present or not in the process. Each element vi of $\mathrm{V}$ is obtained by applying the corresponding decision procedure $\mathrm{i}(\mathrm{n})$, ie vi $=\Phi \mathrm{i}(\mathrm{n})$. Thus, an alarm is generated when vi $=1 \Rightarrow \mathrm{V} /=[00 \ldots 0]$.

Theoretically, if the system works, the value of each residue ri $(i=1, . ., n)$ must be equal to zero. But in practice and in the simplest case, $\mid \Psi \Delta \mathrm{T}$ (ri) $\mid$ is limited by a small amount $\mathrm{i}$ with pretreatment $\Psi \Delta \mathrm{T}$ on each residue (i.e., a running average over a time interval $\Delta \mathrm{T}$ ). The parameter $\mathrm{i}$ is a fixed threshold (by experience, for example). Indeed, in practice, the residual values in normal operation are never zero. This is due to modeling errors and noise measurements. To decide the presence of a failure, each treated residue is compared with a threshold value $i$ set as a priori. In the simplest case, each element vi of $\mathrm{V}$ is obtained by using the following condition:

$v_{i}=\left\{\begin{array}{c}S_{j i}=1, \text { if }\left|\psi_{\Delta T}\left(r_{i}\right)\right|>\epsilon_{i} \\ 0, \text { else }\end{array}\right.$

\section{6) Robust Diagnostic Bond Graph approach6}

a) Representation LFT: Linear Fractional Transformations (LFTs) are very generic objects used in the modeling of uncertain systems. The universality of LFT is because any regular expression can be written in this form. [21] This form of representation is used for the synthesis of an uncertain system of law control according to the principle of $\mu$ analysis. It consists of separating the nominal part of a model from its uncertain part, as shown in Fig. 9. The nominal values are grouped in an augmented matrix denoted M, supposedly clean, and an uncertainty whatever their type (parametric uncertainties, structured and unstructured model uncertainty, measurement noise, etc.) is connected in a matrix structure diagonal $\Delta$.

b) Modeling of Bond Graph uncertain elements: The BG deterministic models of a physical element $\mathrm{R}$ of the causality resistance and the conductance are shown respectively in Fig. 10 (a) and (b).

Bond Graph element with additive uncertainty.

By introducing an uncertainty additively on the element $R$ in causal resistance are obtained:

$e_{R}=\left(R_{n}+\Delta R\right) f_{R}=R_{n} f_{R}+\Delta R f_{R}=e_{n}+e_{i n c}$

$R_{n}$ With the nominal value of the element $R \Delta R$ and the additive uncertainty parameter. $\Delta R$ And $f_{R}$ are respectively the effort and flow in the element in R. Rn.fR = and $=$ EINC $\Delta$ R.fR represent respectively the effort made by the nominal setting and effort introduced by the additive uncertainty. Equation (42), clearly shows that the effort introduced by. $\Delta \mathrm{R}$ additive uncertainty is independent of the nominal value of the parameter $\mathrm{Rn}$. This form of representation is valid for linear systems with constant parameters. The bond graph equivalent model to the mathematical model of equation (42) is given by Fig. 11(a) and Fig. 11(b) shows the bond graph model of the element R in causal conductance. The bond graph approach can anticipate problems simulation and generation of robust RRAS.

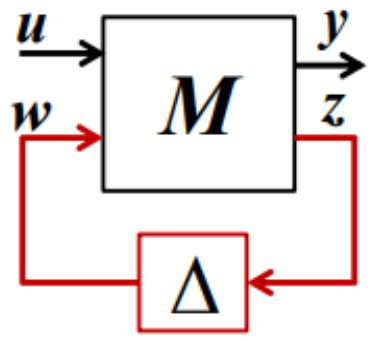

Fig. 9. LFT Representation.

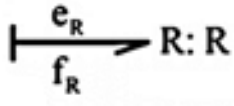

(a)

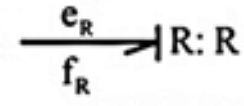

(b)
Fig. 10. The Deterministic Elements of Causal in the Resistance R (a) and the Causal Conductance (b).

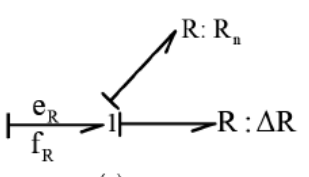

(a)

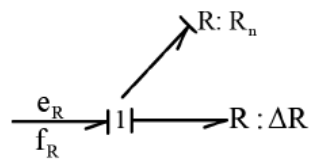

(b)
Fig. 11. Element R Causality Resistance with Additive uncertainty (a). In Causal Conductance (b). 
Indeed, in the case where the uncertainty is introduced additively as a BG member (Bond Graph), the structural properties of the uncertain model prevent the automatic generation of the robust RRAS and generate the errors in the simulated model. To illustrate this feature, the analysis of the BG models and the $\mathrm{C}$ and I elements with integral causality are derived in Fig. 12 and 13.

The BG models of $\mathrm{C}$ and $\mathrm{I}$ in the integral causality presented in Fig. 12(a) and 13(a) show that the uncertainty represented by a link graph element is causally related to the nominal parameter with no link of causality with the rest of the model. This situation creates a differential between the nominal loop element and the uncertainty. Thus, for complex models, where the number of uncertainties parametric is important, the dynamic simulation model is practically impossible because of the algebraic differential loops. When BG models $\mathrm{C}$ and $\mathrm{I}$ elements are derived by causality (Fig. 12(b), 13 and (b)), the nominal parameter $\left(C_{n}\right.$ and $\left.I_{n}\right)$ pass through the causal derivative and the parameter corresponding to the uncertainty ( $\Delta C$ and $\Delta I$ ) pass through the integral causality, but the differential loop is still present.

BG-LFT element with a multiplicative uncertainty

The introduction of a multiplicative uncertainty on the element $\mathrm{R}$ in resistance causality gives:

$e_{R}=R_{n}\left(1+\delta_{R}\right) f_{R}=R_{n} f_{R}+\delta_{R} R_{n} f_{R}=e_{n}+\delta_{R} e_{n}=e_{n}+e_{i n c}$

$R_{n}$ with the nominal value of the element $\mathrm{R}$ and $\Delta R$ Multiplicative uncertainty parameter. $e_{R}$ and $f_{R}$ are respectively the effort and flow in the parameter $\mathrm{R}=\delta_{R} R_{n} f_{R}$ $e_{i n c} R_{n} f_{R}$ and represent the effort made by the nominal setting and effort introduced by the multiplicative uncertainty. Unlike the effort introduced by an additive uncertainty concerning the parameter (eq. (45), the effort made by a multiplicative uncertainty (eq. $(47,45)$ ) is a function of the effort made by the nominal setting.

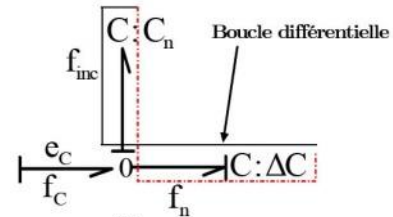

(a)

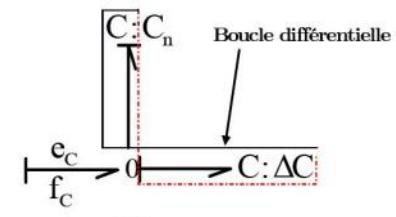

(b)
Fig. 12. The C Element in the Integral Causality with the Additive uncertainty (a). The Causally Derivative (b).

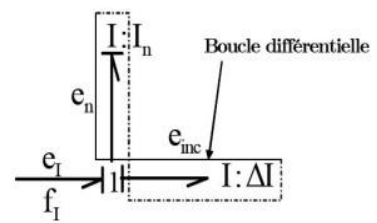

(a)

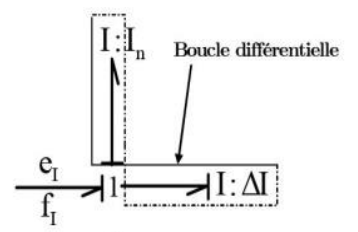

(b)
Fig. 13. Element I in Integral Causality with Additive uncertainty (a). Causally Derivative (b).
With 47 is:

$q(t)=\int^{t} f(t) d t=q_{0}+\int_{t_{0}}^{t} f(t) d t$

The introduction of a multiplicative uncertainty element $\mathrm{R}$ in conductance causality gives:

$$
\begin{aligned}
& f_{R}=\frac{1}{R_{n}}\left(1+\delta_{\frac{1}{R}}\right) e_{R}=\frac{1}{R_{n}} e_{R}+\delta_{\frac{1}{R}} \frac{1}{R_{n}} e_{R} \\
& =f_{n}+\delta_{\frac{1}{R}} f_{n}=f_{n}+f_{\text {inc }}
\end{aligned}
$$

The multiplicative uncertainty on the element $\mathrm{R}$ in causal conductance. $e_{R}$ and $f_{R}$ are respectively the force and the flux in the element $\mathrm{R}$ in causal conductance.

$f_{n}=\frac{1}{R_{n}} e_{R} \quad f_{\text {inc }}=\delta_{\frac{1}{R}} \frac{1}{R_{n}} e_{R}$

Represent respectively the flow provided by the nominal parameter and the flow introduced by the multiplicative uncertainty. The equivalent models of the synoptic scheme of the equations (43) and (45) are respectively given in Fig. (14)(a) and (b).

From equations (45) and (47), we can construct the jump graph model in Fig. 15(a) and (b). Passing by a deterministic BG model in a form of uncertain LFT model, therefore, consists in introducing the modulated sources of effort or flux, which are respectively added to the junctions 1 or 0 . Thus, the model is correct and its structurally causal properties are not modified (controllability, observability, etc.). Moreover, these modulated sources have a causal link with the rest of the model, in which the energy supply is spread in the form of effort or flux and is added to the energetic balance at the junctions. This standard form is suitable for diagnosing systems with uncertain parameters [22].

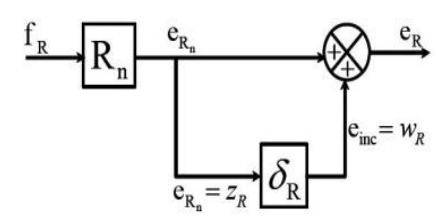

(a)

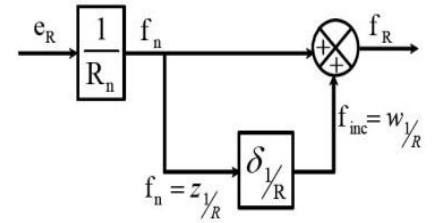

(b)
Fig. 14. LFT Block Diagram Model of a Resistance Element R Causally with Multiplicative uncertainty (a). Causality Conductance (b).

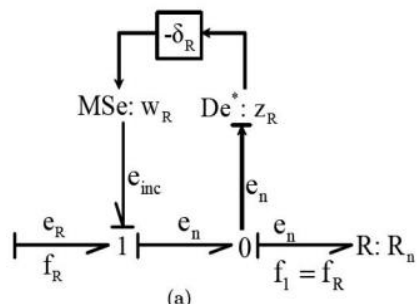

(a)

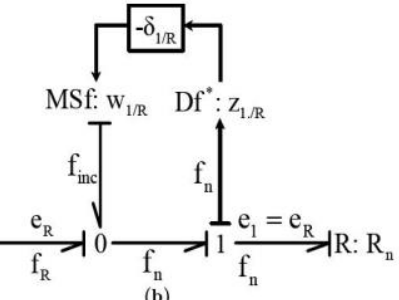

(b)
Fig. 15. The BG-LFT Model and the Causality R Resistance Element with the Multiplicative uncertainty (a) .in Causal Conductance (b). 
c) Construction of a BG-LFT model: This method consists of replacing each element with its uncertain BG-LFT. Full BG-LFT can then be represented by the diagram of Fig. 16 when the standard structure of Fig. 9 appears. On a link graph model, each element corresponds to a specific hardware component or physical phenomenon, thus facilitating the identification of the numerical values of the parameters and uncertainties, and the transition to the LFT form is done by a simple addition modulated sources in the effort and in the model data flow. The state variables of a bond graph model are related to the dynamic elements I and C, and the causal and structural aspects of the model allow the automatic generation of an uncertain state model in the standard 9 format.

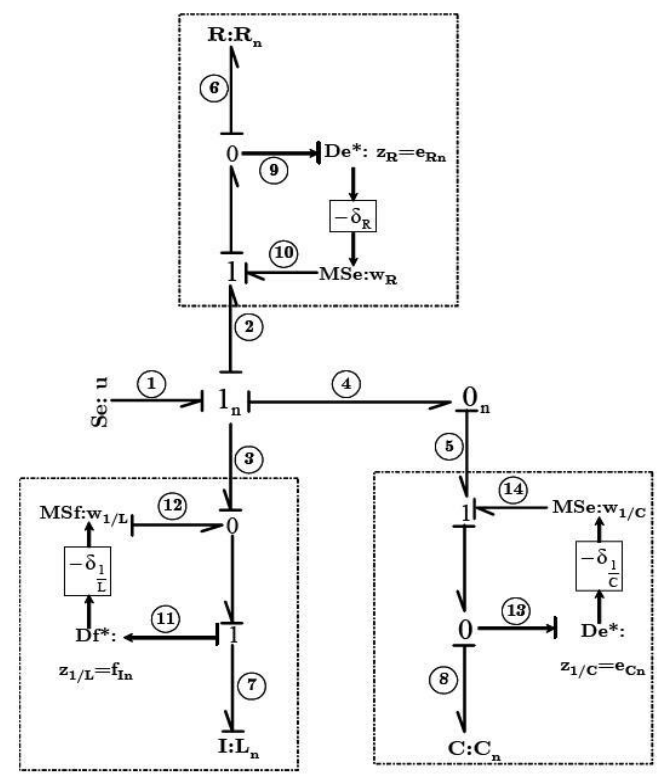

Fig. 16. Representation of a BG-LFT.

Example: Given the RC circuit, I Next (Fig. 17).

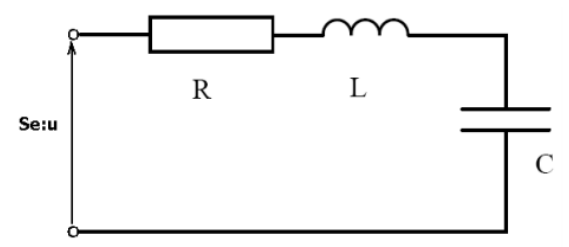

Fig. 17. Electrical Circuit R, C, I.

The deterministic bond graph model of the system is given in Fig. 18.

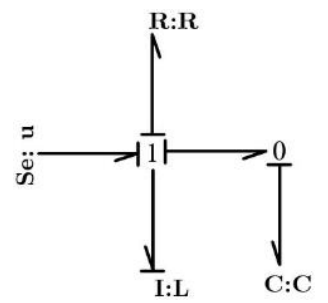

Fig. 18. Deterministic Model BG Integral Causality of a Circuit R, C, I.

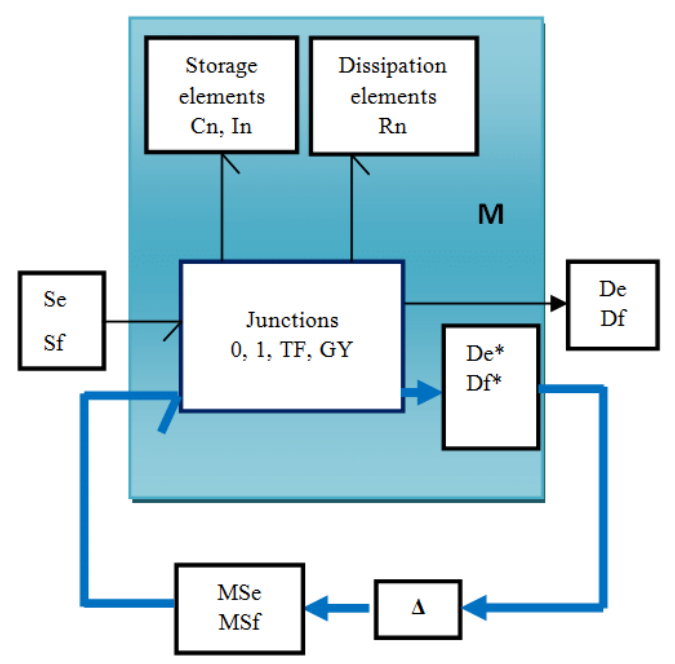

Fig. 19. BG-LFT Model in Integral Causality of a Circuit R, C, I.

The bond graph model uncertain as LFT is obtained by replacing each element by its uncertain BG-LFT model; we obtain the BG-LFT model of Fig. 19. Parametric uncertainties $\Delta R, \delta 1 \mathrm{R}, 1 \delta \mathrm{C}$ corresponds to specific hardware components. Interactions efforts (or flows) introduced by the nominal parameters and uncertainties are performed by the causal and structural constraints of the model; efforts (or flux) are propagated along causal paths and are added to a junction 1 (or a junction 0 ).

7) Generating robust residues:

a) The general form of the uncertain RRA: Generating robust analytic connections redundancy from clean, observable and over determined link graph models is summarized by the following steps:

- Step 1: Verifying the state of coupling with the deterministic causal model of the preferential derivative link graph; if the system is over determined, then, continue the following steps.

- Step 2: The link graph model is formed in LFT.

- Step 3: The symbolic expression of the RRA is deduced from the equation junctions. The first form will be expressed by:

$\sum b_{i} f_{i_{n}}+\sum S f+\sum \omega_{i}=0$

For a junction 0:

$\sum b_{i} e_{i_{n}}+\sum S e+\sum \omega_{i}=0$

For a junction $\Sigma$ Sf 1: With the sum of the sources flows due to the junction $0, \Sigma$ Se the sum of the sources flows to the junction 1 , and $b= \pm 1$ depending on whether the half-arrow into or out of the junction. The unknown variables and ein end.

- Step 4: The unknown variables are eliminated by browsing causal paths between the sensors or sources and unknown variables. 
- Step 5: After removal of the unknown variables, the uncertain RRAS is in the form:

$$
\begin{aligned}
& R R A: \phi\left(\sum S e, \sum S f, D e, D f, \tilde{D} e, \tilde{D} f,\right. \\
& \left.\sum \omega_{i}, R_{n}, C_{n}, I_{n}, T F_{n}, G Y_{N}\right)=0
\end{aligned}
$$

Where, TFn and Gyn are respectively the nominal values of the moduli of TF and GY elements. Rn, $\mathrm{Cn}$ and In are the nominal values of the components R, C and I. $\Sigma$ wi is the sum of the modulated inputs corresponding to the uncertainties on the junction-related items.

b) Generation of adaptive thresholds: The generated RRAS consists of two separated parts by the use of the LFT model, a nominal part denoted $\mathrm{r}$ :

$r=\phi\left(\sum S e, \sum S f, D e, D f, \tilde{D} e, \tilde{D} f, \sum \omega_{i}, R_{n}, C_{n}, I_{n}, T F_{n}, G Y_{N}\right)$

and uncertain part denoted with

$b=\sum \omega_{i}:$

$\omega_{i}=\phi\left(D e, D f, \tilde{D} e, \tilde{D} f, R_{n}, C_{n}, I_{n}, T F_{n}, G Y_{n}, \delta_{R}, \delta_{I}, \delta_{C}, \delta_{T F}, \delta_{G Y}\right)$

Where, $\Delta R \mathrm{dI}, \mathrm{Ac}, \delta \mathrm{TF}, \delta \mathrm{GY}$ are respectively the values of multiplicative uncertainty on the elements R, I, C, TF and GY, the uncertain portion of the RRA used for generating the adaptive thresholds of normal operation as an envelope that contains the residue in the absence of the defects. A parametric uncertainty can be defined as a slight deviation of the parameter from its nominal value, with no effect on the proper functioning of the system. It can be constant or variable and may vary randomly in a positive sense and in a negative direction. Given this kind of uncertainty parameters, and by using the properties of the absolute value of a real:

$\left|\omega_{i}\right|=\left|\delta_{i} e_{i n}\right|$

$\left|\omega_{i}\right|=\left|\delta_{i} f_{\text {in }}\right|$

Indeed starting $R R A$ of equation (50), $\mathrm{r}$ and substituting its value (eq. (53)) in the RRA are obtained:

$r+\sum \omega_{i}=0 \Rightarrow r=-\sum \omega_{i}$

While asking

$a=\sum\left|\omega_{i}\right|$

We then

$r \leq a$

Since the variation of the residue is the image of the variation of the uncertainties in the absence of defects, it can vary in a positive direction and a negative direction. It is, therefore, necessary to generate a lower (negative) threshold a noted.

$-a \leq r \leq a$

In this part, we presented the diagnostic methods according to the Bond Graph approach, the use of observers with unknown inputs does not provide a representation of the fault deduced directly from the model of the link graph. The analytic redundancy relationships generated using the parity space method depends on knowing the degree of diversion to be applied. The benefits of using this latter method consist of the simplicity of understanding the (RRAS) because they correspond to the relationships and the variables displayed by the bond graph model, then the transition to the LFT form by simply adding the data source. The effort and the flow modulated the image of the physical process.

\section{APPLICATION AND SIMULATION RESUlts}

The previous sections have presented the diagnostic methods based on the link graph models. In this section, we will illustrate some examples of technical applications based on the generation of analytic redundancy relationships. The system in Fig. 20 represents the diagnostic techniques of academic platform tests. It consists of 3 tanks, 3 valves and a flow source.

The jump pattern corresponding to the graph is given in Fig. 21.

The analytical redundancy relations generated from the model in Fig. 21 are given by:

$$
A R R_{1}=s_{f}-\phi c_{T 1}(P)-\phi_{R_{V 1}}\left(P_{1}, P_{2}\right)=0
$$

With

$$
\begin{aligned}
& \phi c_{T 1}\left(P_{1}\right)=\frac{A_{1}}{g} \frac{d P_{1}}{d t} \\
& \phi_{R_{V 1}}\left(P_{1}, P_{2}\right)=C_{d b} \cdot \operatorname{sign}\left(P_{1}-P_{2}\right) \sqrt{\left|P_{1}-P_{2}\right|} \\
& A R R_{2}=\phi_{R_{V 1}}\left(P_{1}, P_{2}\right)-\phi c_{T 2}\left(P_{2}\right)-\phi_{R_{V 2}}\left(P_{2}, P_{3}\right)=0
\end{aligned}
$$

With

$$
\begin{aligned}
& \phi c_{T 2}\left(P_{2}\right)=\frac{A_{2}}{g} \frac{d P_{2}}{d t} \\
& \phi_{R_{V 2}}\left(P_{2}, P_{3}\right)=C_{d b} \cdot \operatorname{sign}\left(P_{2}-P_{3}\right) \sqrt{\left|P_{2}-P_{3}\right|} \\
& A R R_{3}=\phi_{R_{V 2}}\left(P_{2}, P_{3}\right)-\phi c_{T 3}\left(P_{3}\right)-\phi_{R_{V 3}}\left(P_{3}\right)=0
\end{aligned}
$$

With

$$
\begin{aligned}
& \phi c_{T 3}\left(P_{3}\right)=\frac{A_{3}}{g} \frac{d P_{3}}{d t} \\
& \phi_{R_{V 3}}\left(P_{3}\right)=C_{d o} \cdot \operatorname{sign}\left(P_{3}\right) \sqrt{\left|P_{3}\right|}
\end{aligned}
$$

The three residues represented in Fig. 22 are the result of the introduction of a fault type "leak" $\mathrm{t}=0.25 \mathrm{t}=0.75$ at the No. 1 tank.

The introduction of the same type of defect in No. 2 tank gives the results in Fig. 23

The same fault as the previous two results is introduced into vessel 3, giving the residues represented by Fig. 24: 
The result of a combined defect of the three tanks at the same time is given in Fig. 25.

The response of the system in the presence of three simultaneous faults is shown in Fig. 26.

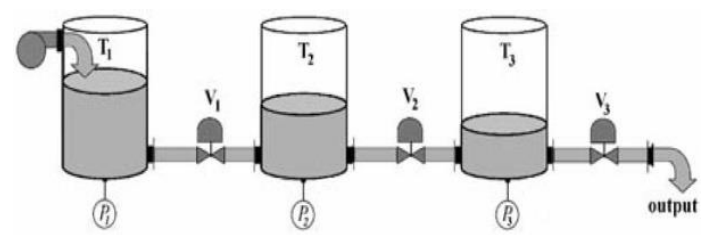

Fig. 20. Hydraulic System 3 Tanks.

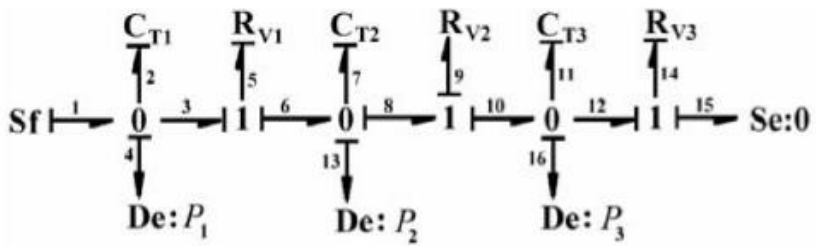

Fig. 21. Hydraulic System Model Bond Graph 3 Tanks.

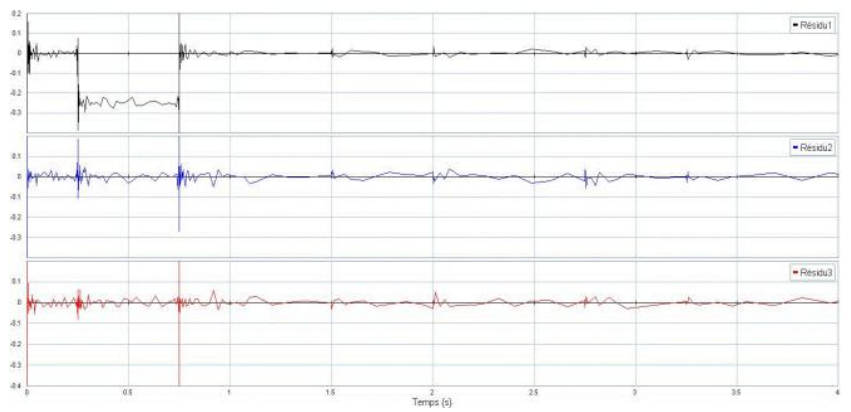

Fig. 22. Residues Resulting from a Defect Leakage on Tank 1.

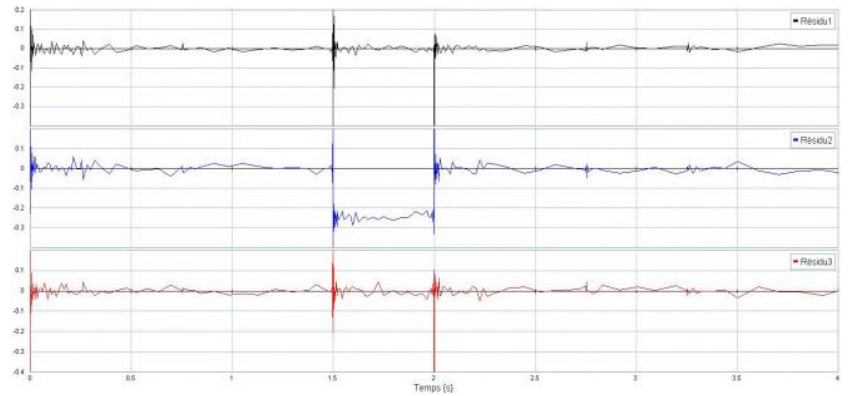

Fig. 23. Residues Resulting from a Defect Leakage on the Tank 2.

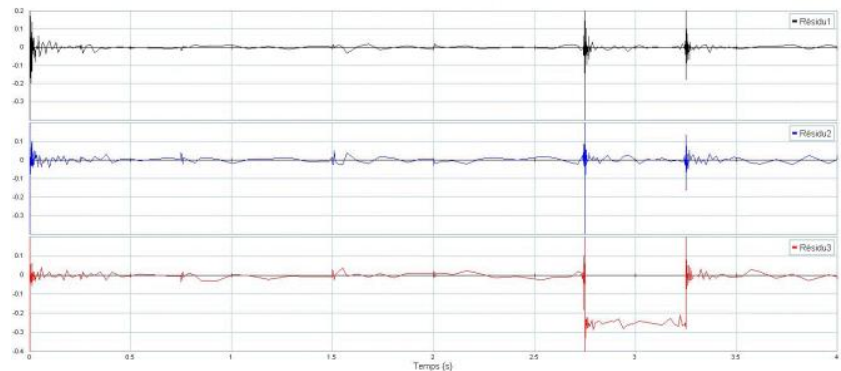

Fig. 24. Residues Resulting from a Defect Leakage on the Tank 3.

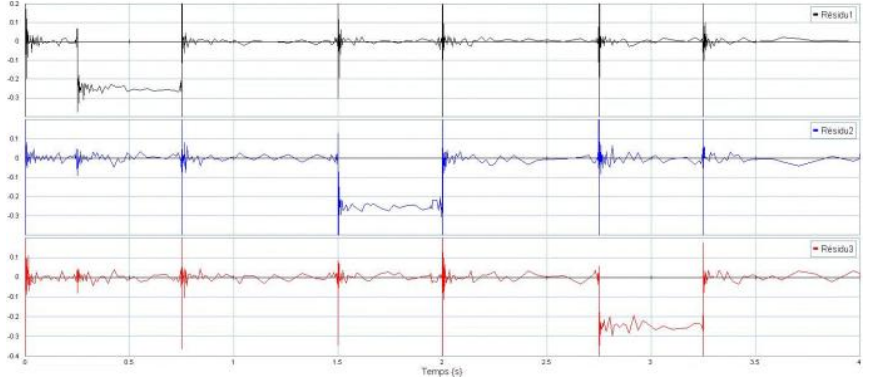

Fig. 25. Bottoms Combined Defects on the Tank 1.2 and 3.

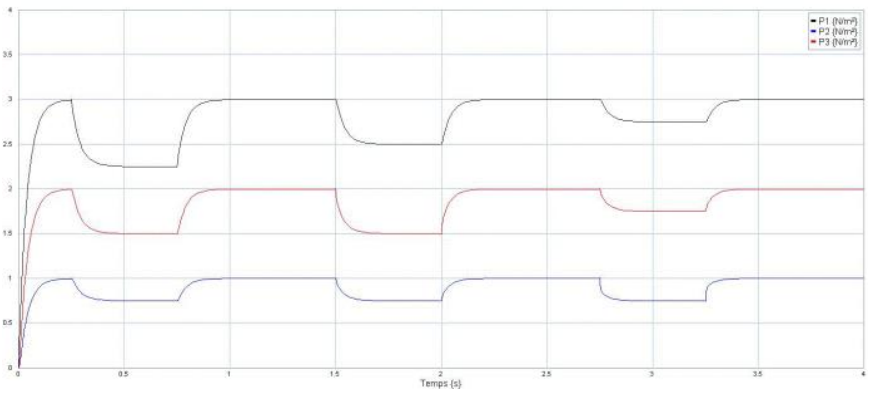

Fig. 26. Response System to Three Defects Combined on the Tanks 1, 2 and 3 .

\section{DISCUSSIONS}

The results of the application of the basic diagnostic method to the bond graph model proved the effectiveness of the technique in detecting the insulation defects in a single system.

- For the detection: the residue is zero as long as there is no fault in the system, the introduction of a default passed the residue to a non-zero value.

- For the isolation: the passage of the residue at a non-zero value (or exceeds the threshold) is the result of a defect in the corresponding tank.

1) Case study of an asynchronous motor

a) Bond Graph Model of the asynchronous motor: The bond graph model of the asynchronous squirrel-cage motor is given by Fig. 27.

The simulation model presented above shows the obtained results (ias, ibs, ics and angular velocity) (Fig. 28).

The load torque absence is shown in Fig. 29:

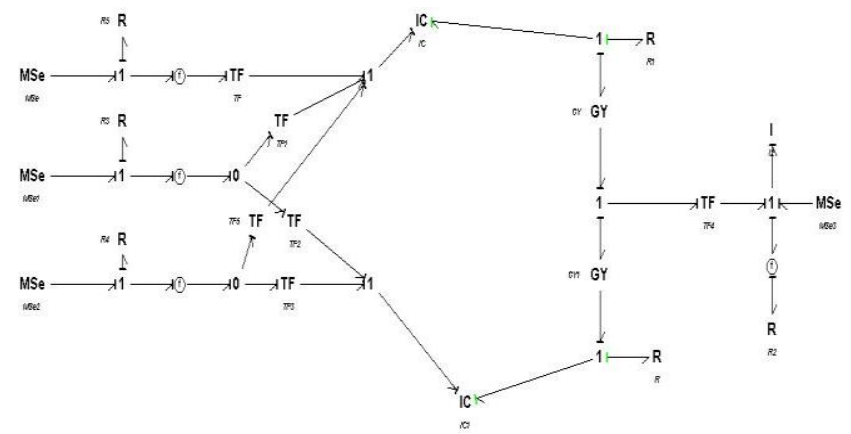

Fig. 27. Bond Graph Model of the Asynchronous Motor with Cage. 


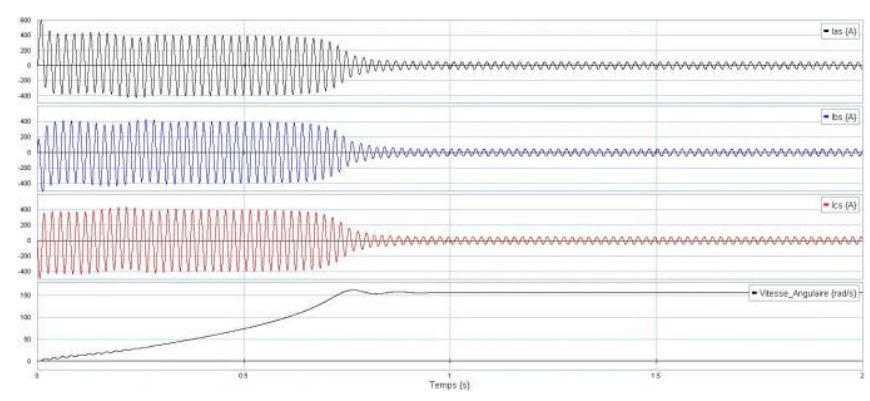

Fig. 28. The Stator Currents and the Angular Velocity of the MAS.

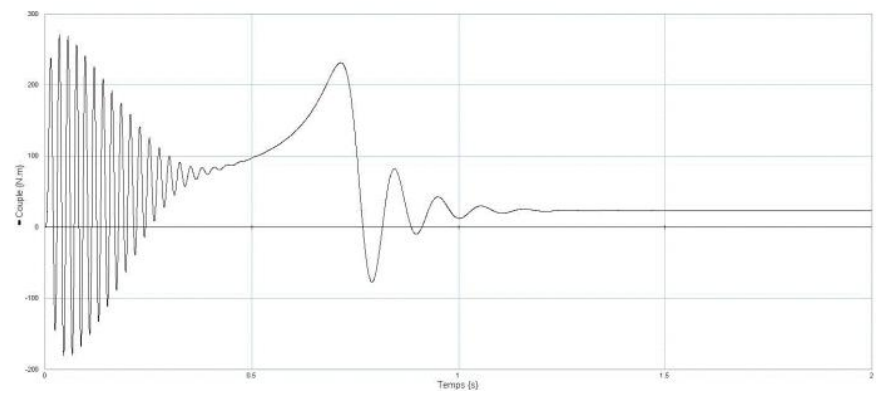

Fig. 29. The Couple Absence Charge.

The torque as a function of the angular velocity is illustrated in Fig. 30.

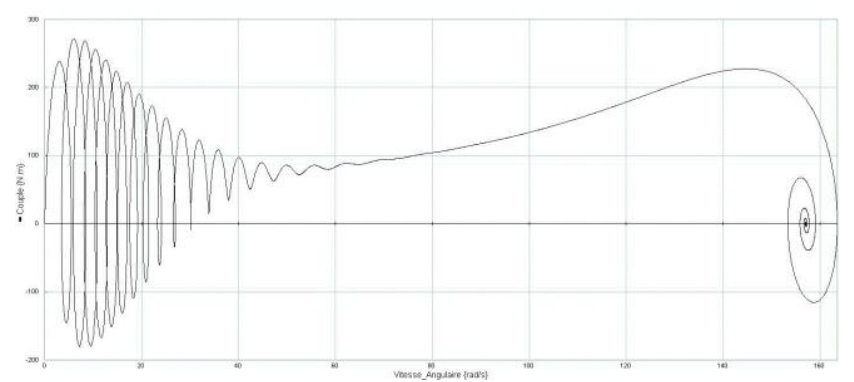

Fig. 30. The Torque Depending on the Angular Velocity of Load Absence.

The development of the asynchronous motor modeling step is detailed in [23].

However, to introduce the defects in the rotor of the asynchronous motor, it is necessary to represent the expression of the currents in each bar. The link graph model presented in the following section will allow us to apply the diagnostic methods studied in the previous sections

b) BG model of an asynchronous motor with cage for the diagnosis: The bond graph model presented in Fig. 31 will allows us to introduce the defects (bar Break, shorted turns in a coil, ...) in the asynchronous motor. (The modeling step is detailed in [23], [24] and [25]).

The stator currents in the two-phase mark are:

$\left[\begin{array}{l}i_{\alpha} \\ i_{\beta}\end{array}\right]=\left[\begin{array}{ccc}\frac{\sqrt{2}}{\sqrt{3}} & -\frac{1}{\sqrt{6}} & -\frac{1}{\sqrt{6}} \\ 0 & \frac{1}{\sqrt{2}} & -\frac{1}{\sqrt{2}}\end{array}\right]\left[\begin{array}{l}i_{s a} \\ i_{s b} \\ i_{s c}\end{array}\right]=\left[\begin{array}{ccc}\frac{1}{m_{1}} & \frac{1}{m_{2}} & \frac{1}{m_{3}} \\ 0 & \frac{1}{m_{4}} & -\frac{1}{m_{5}}\end{array}\right]\left[\begin{array}{l}i_{s a} \\ i_{s b} \\ i_{s c}\end{array}\right]$

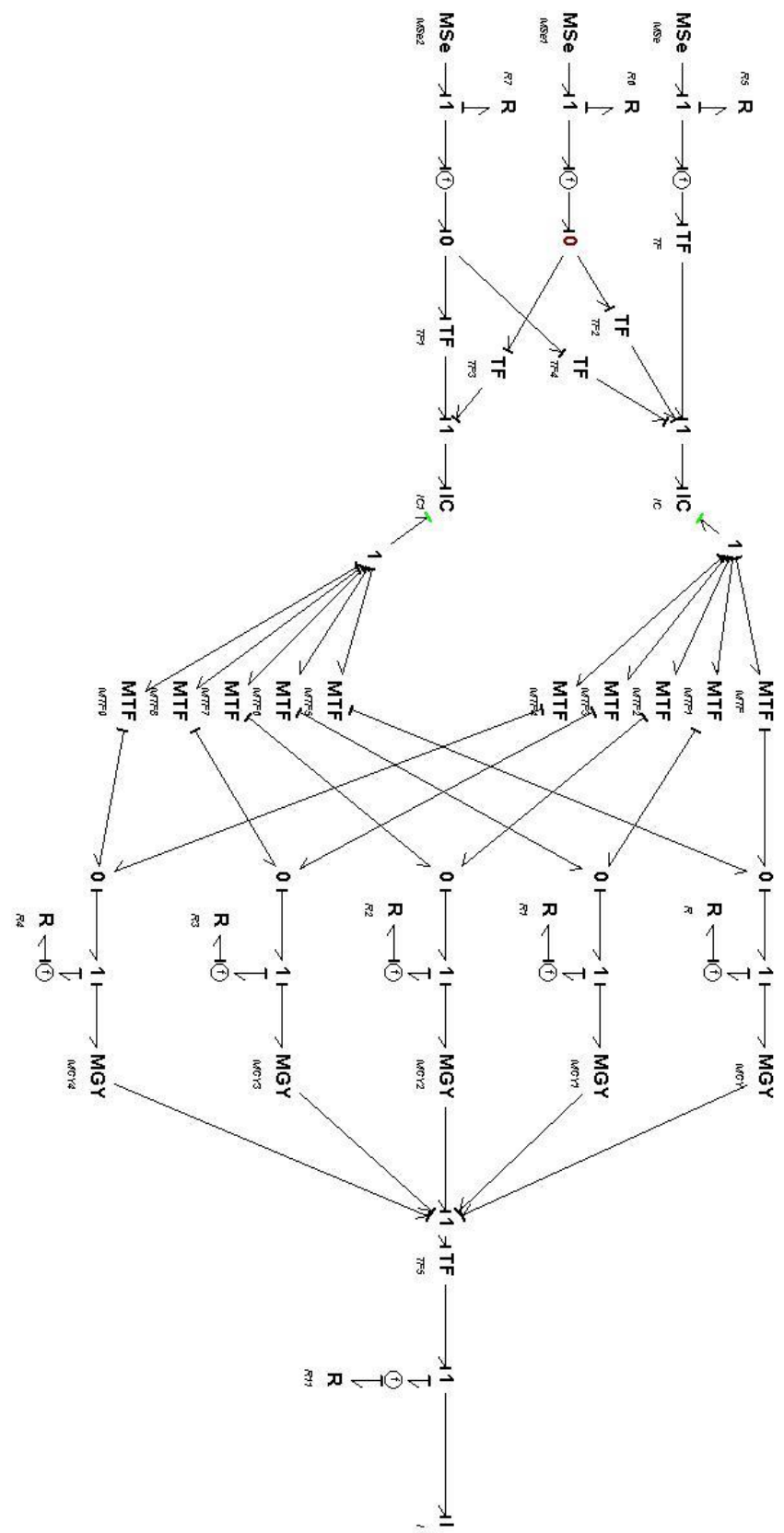

Fig. 31. BG Model of the Asynchronous Motor for the Diagnosis.

The stator voltages are given by the following equations:

$\left[\begin{array}{c}V_{\alpha s} \\ V_{\beta s} \\ 0 \\ 0\end{array}\right]=\left[\begin{array}{cccc}R_{s}+\frac{L_{s} d}{d t} & 0 & \frac{L_{m} d}{d t} & 0 \\ 0 & R_{s}+\frac{L_{s} d}{d t} & 0 & \frac{L_{m} d}{d t} \\ \frac{L_{m} d}{d t} & L_{m} \omega_{r} & R_{r}+\frac{L_{r} d}{d t} & L_{r} \omega_{r} \\ -L_{m} \omega_{r} & \frac{L_{m} d}{d t} & -L_{r} \omega_{r} & R_{r}+\frac{L_{r} d}{d t}\end{array}\right]\left[\begin{array}{c}i_{\alpha s} \\ i_{\beta s} \\ i_{\alpha r} \\ i_{\beta r}\end{array}\right]$

The electromagnetic torque for an induction motor with pole $\mathrm{P}$ is given by.

$$
T_{e}=\frac{P}{2}\left[i_{\alpha r}\left(L_{m} i_{\beta s}+L_{r} i_{\beta r}\right)-i_{\beta r}\left(L_{m} i_{\alpha s}+L_{r} i_{\alpha r}\right)\right]
$$


The mechanical equation is:

$T_{e}=J \frac{d \omega}{d x}+c \omega+T_{L}$

Such as:

The moment of inertia is given by $\mathrm{J}=0.4 \mathrm{~kg} \mathrm{~m} 2 \cdot \mathrm{c}$ the friction of the shaft bearings is $+=0.15 \mathrm{~N} \cdot \mathrm{s} / \mathrm{m}$ load torque TL. V $\alpha$ s, V $\beta$ s are the stator voltages of the $\alpha$ and $\beta$ axes in the Park coordinate system. i $\alpha$ s and $i \beta$ s are the stator currents of the $\alpha$ and $\beta$ axes in the Park coordinate system. i $\alpha$, i $\beta r$ are the rotor currents of the $\alpha$ and $\beta$ axes in the Park coordinate system. Rs is the stator resistance $0.0788 \Omega \operatorname{Rr} 1 \ldots \operatorname{Rr} 5$ bars are the Rotor resistors $0.0408 \Omega \mathrm{Ls}=$ stator inductance $=0$.

The equation characterizing the IC element of Fig. 31 is given by:

$\phi_{I C}=\left[\begin{array}{c}\lambda_{\alpha s} \\ \lambda_{\alpha r}\end{array}\right]=\left[\begin{array}{cc}L_{s} & L_{m} \\ L_{m} & L_{r}\end{array}\right]\left[\begin{array}{l}i_{\alpha s} \\ i_{\alpha r}\end{array}\right]$ [23]

Expression of the currents in each rotor bar is as follows:

$i_{r k}=m\left[i_{\alpha r} \cos \left\{\theta+\frac{2(k-1) \pi}{n}\right\}+i_{\beta r} \sin \left\{\theta+\frac{2(k-1) \pi}{n}\right\}\right]$

Such as:

$m=\sqrt{\frac{2}{n}}$

We ask:

$m r_{k}=m * \cos \left\{\theta+\frac{2(k-1) \pi}{n}\right\}$

$k=1,2, \ldots, n$

$m r_{k+n}=m * \sin \left\{\theta+\frac{2(k-1) \pi}{n}\right\}$

$k=1,2, \ldots n$

whith

$n=5$

The $m r_{k}$ y represents the modulated Transformer Modules revealed in Fig. 31. By substituting the expression of the currents in the electromagnetic torque equation (57):

$T_{e}=\sum_{k=1}^{5} T_{k}=\frac{P}{2} \sum_{k=1}^{5} \frac{\sqrt{2}}{\sqrt{5}}\left[\lambda_{\beta r} \cos \left(\theta+\frac{2(k-1) \pi}{n}\right)-\lambda_{a r} \sin \left(\theta+\frac{2(k-1) \pi}{n}\right)\right] i_{r k}$

We ask:

$r_{k}=\sqrt{\frac{2}{n}}\left[\lambda_{\beta r} \cos \left(\theta+\frac{2(k-1) \pi}{n}\right)-\lambda_{a r} \sin \left(\theta+\frac{2(k-1) \pi}{n}\right)\right]$ $r_{k}$ Represent the modules gyrators $M G Y M G Y \rightarrow 4$ in Fig. 31 the stator currents and the angular velocity are depicted in Fig. 32.

The rotor current of the induction motor healthy cage is shown in Fig. 33.

c) Asynchronous engine diagnostics: The application of the method presented in the previous section on the model shown in Fig. 31 results in the generation of the following analytical redundancy relations:

$R A A_{1}=V_{a}-\phi_{R_{s a}}-m_{1} *\left(\phi_{I 1}+\frac{1}{m_{2}}\left(V_{b}-\phi_{R_{s b}}\right)-\frac{1}{m_{3}}\left(V_{c}-\phi_{R_{s c}}\right)\right)=0$
$R A A_{2}=\phi_{I 2}+\phi_{1}+\phi_{2}+\phi_{3}+\phi_{4}+\phi_{5}=0$

With:

$\phi_{I C}=\left[\begin{array}{l}\phi_{I 1} \\ \phi_{I 2}\end{array}\right]$

$\phi_{i}=m_{r i}\left(\phi_{R r i}+\frac{r_{i}}{m_{6}} \omega\right)$

Residues of an asynchronous motor without fault are shown in Fig. 34.

A bar breakage at the instant $(\mathrm{t}=1.5 \mathrm{~s})$ on the rotor of the asynchronous squirrel-cage motor produces the following residues (Fig. 35).

The rotor currents are shown in Fig. 36.

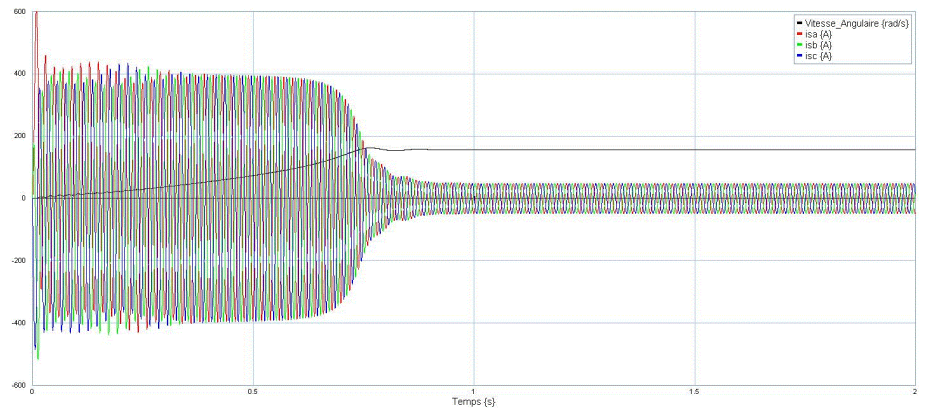

Fig. 32. Stator Currents and Speed of the Asynchronous Motor Healthy.

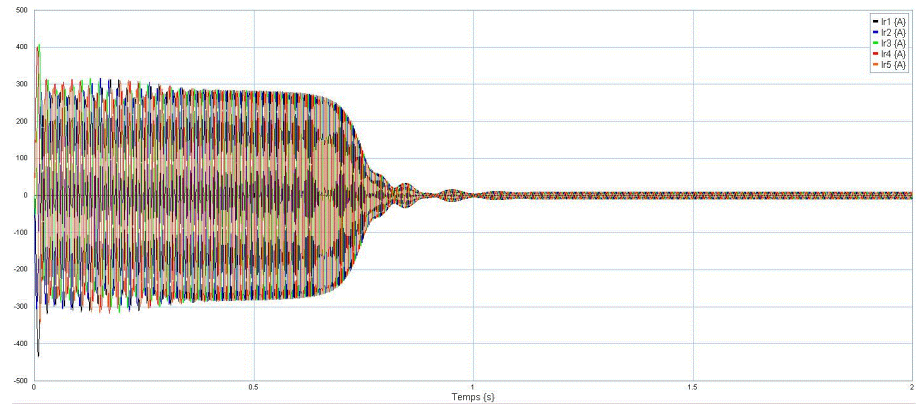

Fig. 33. Rotor Asynchronous Motor Currents of the Healthy. 


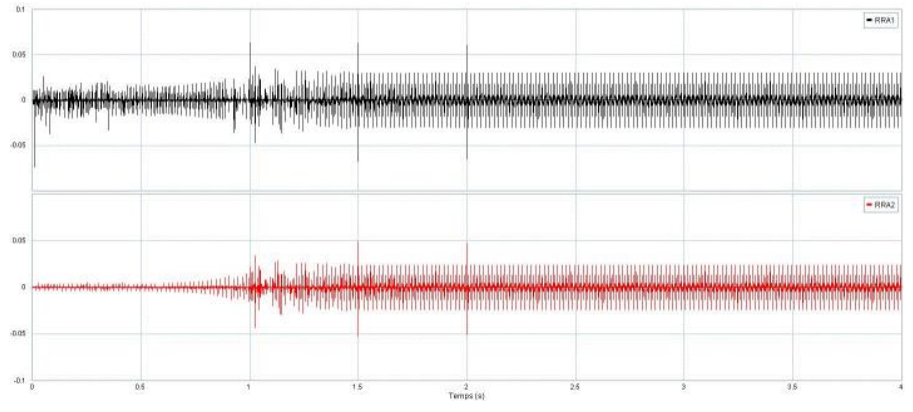

Fig. 34. Residues of a Healthy Asynchronous Motor.

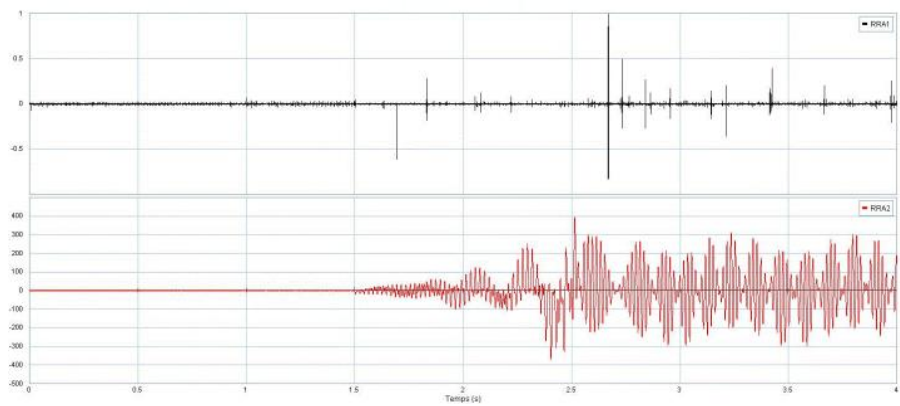

Fig. 35. Residues of an Asynchronous Motor with One Broken Bar.

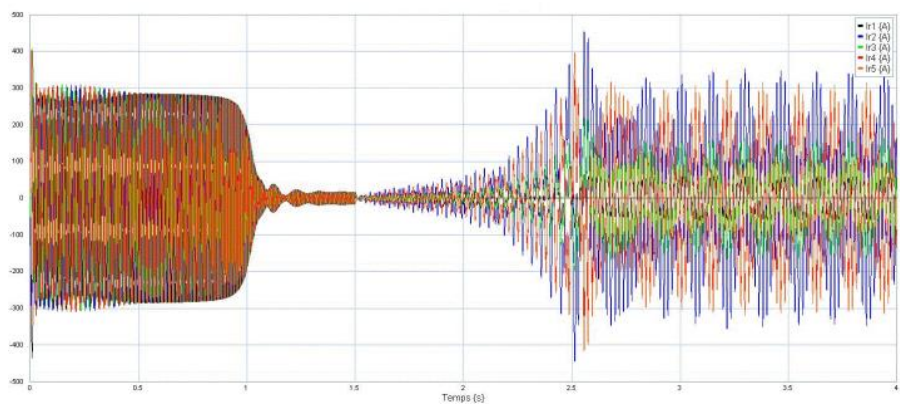

Fig. 36. The Rotor Currents of an Asynchronous Motor with One Broken Bar.

A short circuit turns to $(\mathrm{t}=1.5 \mathrm{~s})$ of a stator coil produces the residues represented by Fig. 37.

An asynchronous motor fault is detected by passing the residues obtained from the RRAS to a non-zero value. The isolation is achieved by the sensitivity of each RRAS to a particular fault. A rotor failure (bar breakout) is isolated bypassing the residual 2 (RRA2) to a non-zero value (Fig. 35). A stator fault (short circuit) is isolated from the residue 1 (RRA1) at a value other than zero (Fig. 37).

d) Robust diagnostic asynchronous motor with cage: The uncertain Bond Graph model of an asynchronous motor with a cage is shown in Fig. 38 .

A deviation in the value of the resistance of a stator coil causes a variation of the residue 2, the alarm is not triggered until there is no excess of the value of the adaptive thresholds (士 a) (Fig. 39).

The detection of the index $\mathrm{D}$ allows us later to detect the defect. A short circuit in a stator coil is detected and isolated bypassing the detection index D to a positive value (Fig. 40).

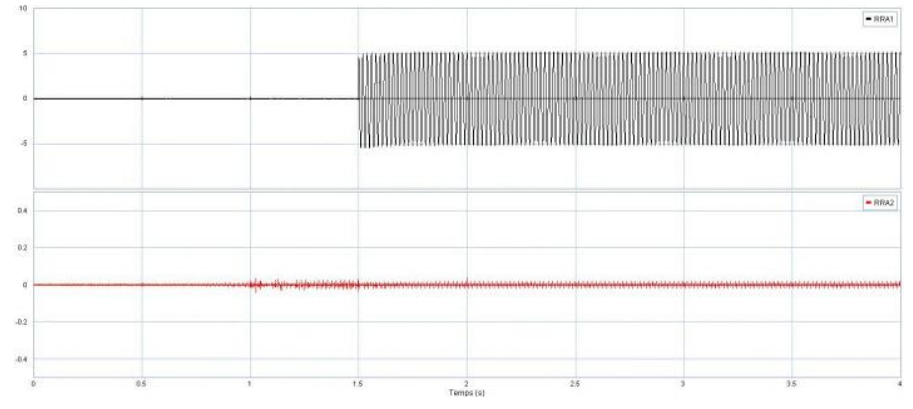

Fig. 37. Residues with Short Circuit the Turns of a Stator Winding.

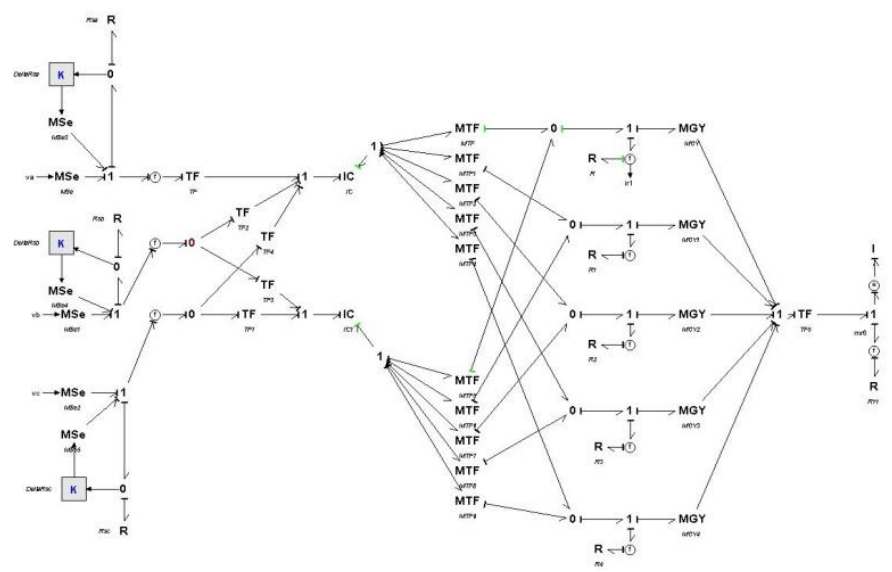

Fig. 38. BG-LFT Model of Asynchronous Motor with Cage.

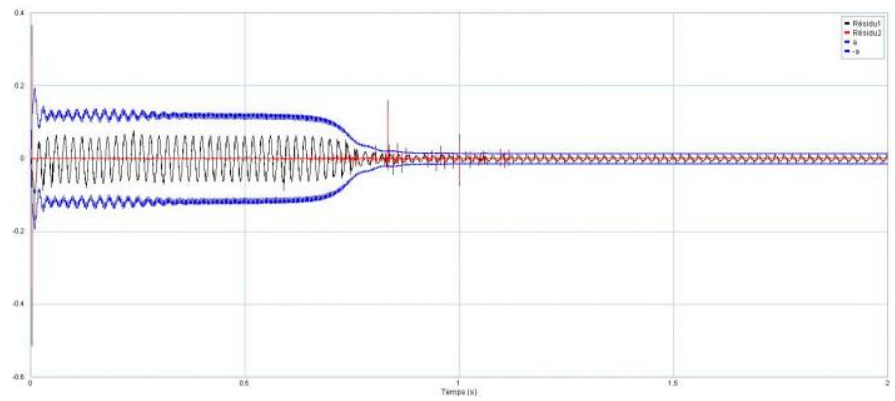

Fig. 39. Residues Robust to Parameter Variations.

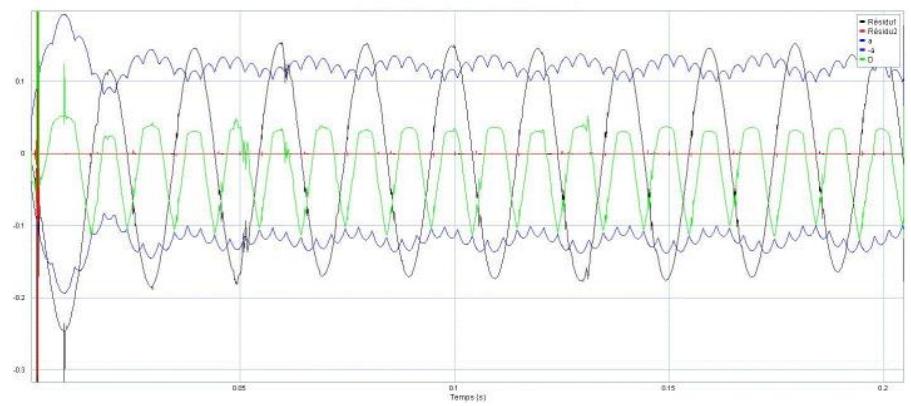

Fig. 40. Fault Detection in the Presence of Parametric Variations.

This section presents the BG LFT modeling and the reliable diagnosis of a cage asynchronous motor. The interaction of different phenomena is taken into account through the energetic properties of the link graph tool. The use of the LFT form to model the link graph elements is 
generated, from the model, the residuals and the adaptive thresholds for normal operation. The detection index is used to detect the defects with the parametric variations of the support in the monitored system.

\section{CONCLUSION}

In the current economic climate, business performance must be growing. For that, the pumping system must always be produced with good performance, with low cost, and inefficient severe security conditions. Also, the processes are increasingly complex and increasingly computerized. Thus, it is less obvious or intuitive to know if everything is going well in a process. For this purpose, monitoring methods that allow the detection and diagnosis of faults (errors) are presented. Over a fault is detected early and correctly diagnosed, the production process will comply with the requirements of the safety requirements. We presented a method that allows a robust diagnostic link graph to include it in a single tool: the modeling and the diagnostics.

There are many perspectives for this research work. Diagnosis wise, complex systems are usually no linear and no stationary, which makes developing diagnosis algorithms that are capable of covering most of the operation ranges very difficult.

Robust diagnosis of no stationary systems is a perspective theme that can be developed with multi-model approach. The difficulty in such approaches lies in the discontinued transition between models which can trigger false alarms.

\section{REFERENCES}

[1] H.Belgacem. Simulation and Modeling of a Photovoltaic Pumping Installation by Bond Graph, indjst, Indian, 2018.

[2] B. Dubuisson. Diagnosis and Recognition Of Shapes. Hermes, Paris, 1990.

[3] S. Gentile. artificial intelligence for monitoring continuous processes. In Proceedings of Summer School On Auto Grenoble, Grenoble, France, 26 September 1996.

[4] R. Iserman. Process modeling is based on fault detection and estimation methods. A Survey Automatica, 20 (4): 387-404, 1984.

[5] Frank PM. Fault diagnosis in dynamic systems using analytical and knowledge-based redundancy - a survey and some new results. Automatica, 26 (3): 459-474, 1990.

[6] J. Ragot and D. Maquin. An algorithm for redundancy Obtaining equation of LTI systems. Automatica, 30 (3): 537-542, 1994.

[7] D. Maquin AND J. Ragot. Diagnosis Of Linear Systems. Hermes, Paris, France, Hermes edition 2000.
[8] R. and B. Iserman Freyermuth. Process Fault Diagnosis Based on Process Model Knowledge-Part I: Principle For Fault Diagnosis With Parameter Estimation. Transaction of the ASME, 113: 620-626, 1991.

[9] DG Luenberger, An Introduction to Observers. IEEE Transactions on Automatic Control. Flight AC-16, N6, pp 596-602, December 1971.

[10] AS Willsky. A Survey of Design Methods for Failure Detection in Dynamic Systems. Automatica, Vol 12, pp 601-611, 1976.

[11] RV Beard. Failure Accommodation in Linear Systems Through SelfReorganization. Ph.D. dissertation, MIT, Dep Aero Astro Cambridge, February 1971.

[12] PJ Feenstra, PJ Masterman G. Biswas, PC Breedveld, Bond graph modeling procedures for fault detection and isolation of complex flow processes, in Proc. ICBGM'01, Simulation Series, Vol. 33, no. 1, 2001, pp. 77-82, ISBN: 1-56555-103-6.

[13] T. Kohda H. Katsube H. Fujihara and K. Inoue. Identification of system failure causes using bond graph models. International Conference on Systems, Man and Cybernetics. Conference Proceedings, 5: 269-274, 1993.

[14] C. Ghiaus. Fault diagnosis of air conditioning systems is based qualitative bond graph. Energy and buildings, 30: 221-232, 1999.

[15] P. Haves, T. Salsbury and J. Wright. Condition monitoring in HVAC sub-systems using first-principles models. ASHRAE Transactions (Part 1), pages 519-527, 1996.

[16] W. Lee, C. and G. Kelly Park. Fault detection in an air-handling unit using residual and recursive parameter identification methods. ASHRAE Transactions (Part 1), pages 528-539, 1996.

[17] S. F. Lin et A. P. Wang. Design of Observers with unknown inputs using eigenstructure assignement. International Journal of Systems Science, 31(6): 705-711,2000.

[18] Mr. Blanke, Mr. Kinnaert, Lunze J., and M. Staroswiecki. Diagnosis and Fault Tolerant Control. Springer-Verlag, 2003.

[19] G. Dauphin-Tanguy, A. Rahmani, and C. Sueur. Bond graph aided the design of controlled systems. Simulation Practice and Theory, 7(5-6): 493-513, 1999.

[20] Mr. Basseville and IV Nikiforov. Detection of Abrupt Changes: Theory and Application. Prentice Hall, ISBN 0-13-126780-9, 1993.

[21] A. Oustaloup. (1994). Robustness. Hermes ISBN. 2-86601-442-1.

[22] A. Djeziri. Diagnosis of uncertain systems by the bond graph approach. Ph.D. thesis of the University of Science and Technology of Lille, December 17, 2007.

[23] J. Kim and Bryant. Bond graph model of a squirrel cage induction motor directly with a physical connection, ASME Journal of Dynamic Systems, Measurement, and Control, Vol. 122, pp. 461-469, September 2000.

[24] B. Umesh Rai L. Umanand. Bond graph model of doubly fed induction three-phase motor using the Axis Rotator element for frame processing. Simulation Modeling Practice and Theory Volume 16, Issue 10, November 2008, Pages 1704-1712.

[25] B. Umesh Rai L. Umanand. Generalized bond graph model of a rotating machine. International Journal of Power Electronics 2009 - Vol.1, No.4 pp. 397-413. 